\title{
AN INFRARED CENSUS OF DUST IN NEARBY GALAXIES WITH SPITZER (DUSTINGS). I. OVERVIEW
}

\author{
Martha L. Boyer ${ }^{1,2}$, Kristen B. W. McQuinn ${ }^{3}$, Pauline Barmby ${ }^{4}$, Alceste Z. Bonanos ${ }^{5}$, Robert D. Gehrz ${ }^{3}$, \\ Karl D. Gordon ${ }^{6}$, M. A. T. Groenewegen ${ }^{7}$, Eric Lagadec ${ }^{8}$, Daniel Lennon ${ }^{9}$, Massimo Marengo $^{10}$, Margaret MeiXner $^{6}$, \\ Evan Skillman $^{3}$, G. C. Sloan ${ }^{11}$, George Sonneborn ${ }^{1}$, Jacco Th. van Loon ${ }^{12}$, And Albert Zijlstra ${ }^{13}$ \\ ${ }^{1}$ Observational Cosmology Laboratory, Code 665, NASA Goddard Space Flight Center, Greenbelt, MD 20771, USA; martha.boyer@ nasa.gov \\ 2 Oak Ridge Associated Universities (ORAU), Oak Ridge, TN 37831, USA \\ ${ }^{3}$ Minnesota Institute for Astrophysics, School of Physics and Astronomy, 116 Church Street SE, University of Minnesota, Minneapolis, MN 55455, USA \\ ${ }^{4}$ Department of Physics and Astronomy, University of Western Ontario, London, ON, N6A 3K7, Canada \\ ${ }^{5}$ IAASARS, National Observatory of Athens, GR-15236 Penteli, Greece \\ ${ }^{6}$ STScI, 3700 San Martin Drive, Baltimore, MD 21218, USA \\ ${ }^{7}$ Royal Observatory of Belgium, Ringlaan 3, B-1180 Brussels, Belgium \\ ${ }^{8}$ Laboratoire Lagrange, UMR7293, Univ. Nice Sophia-Antipolis, CNRS, Observatoire de la Côte d'Azur, F-06300 Nice, France \\ ${ }^{9}$ ESA-European Space Astronomy Centre, Apdo. de Correo 78, E-28691 Villanueva de la Cañada, Madrid, Spain \\ ${ }^{10}$ Department of Physics and Astronomy, Iowa State University, Ames, IA 50011, USA \\ ${ }^{11}$ Astronomy Department, Cornell University, Ithaca, NY 14853-6801, USA \\ 12 Astrophysics Group, Lennard-Jones Laboratories, Keele University, Staffordshire ST5 5BG, UK \\ ${ }^{13}$ Jodrell Bank Centre for Astrophysics, Alan Turing Building, University of Manchester, Manchester M13 9PL, UK \\ Received 2014 August 30; accepted 2014 November 14; published 2014 December 24
}

\begin{abstract}
Nearby resolved dwarf galaxies provide excellent opportunities for studying the dust-producing late stages of stellar evolution over a wide range of metallicity $(-2.7 \lesssim[\mathrm{Fe} / \mathrm{H}] \lesssim-1.0)$. Here, we describe DUSTiNGS (DUST in Nearby Galaxies with Spitzer): a 3.6 and $4.5 \mu \mathrm{m}$ post-cryogen Spitzer Space Telescope imaging survey of 50 dwarf galaxies within $1.5 \mathrm{Mpc}$ that is designed to identify dust-producing asymptotic giant branch (AGB) stars and massive stars. The survey includes 37 dwarf spheroidal, 8 dwarf irregular, and 5 transition-type galaxies. This near-complete sample allows for the building of statistics on these rare phases of stellar evolution over the full metallicity range. The photometry is $>75 \%$ complete at the tip of the red giant branch for all targeted galaxies, with the exception of the crowded inner regions of IC 10, NGC 185, and NGC 147. This photometric depth ensures that the majority of the dust-producing stars, including the thermally pulsing AGB stars, are detected in each galaxy. The images map each galaxy to at least twice the half-light radius to ensure that the entire evolved star population is included and to facilitate the statistical subtraction of background and foreground contamination, which is severe at these wavelengths. In this overview, we describe the survey, the data products, and preliminary results. We show evidence for the presence of dust-producing AGB stars in eight of the targeted galaxies, with metallicities as low as $[\mathrm{Fe} / \mathrm{H}]=-1.9$, suggesting that dust production occurs even at low metallicity.
\end{abstract}

Key words: galaxies: dwarf - galaxies: photometry - galaxies: stellar content - infrared: stars - Local Group stars: AGB and post-AGB - stars: mass-loss - stars: winds, outflows

\section{INTRODUCTION}

\subsection{Dust Production by Evolved Stars}

Intermediate-mass $\left(1 M_{\odot} \lesssim M \lesssim 8 M_{\odot}\right)$ and massive $\left(\gtrsim 8 M_{\odot}\right)$ evolved stars are drivers of galaxy chemical enrichment and evolution via the return of significant amounts of gas and dust to the interstellar medium (ISM). This stellar mass loss also drives the subsequent evolution of the stars themselves. However, post-main-sequence stellar evolution is poorly understood, especially in the short-lived dust-producing phases. Also, it is unclear how the galactic environment (especially metallicity) affects stellar dust production and evolution. DUST in Nearby Galaxies with Spitzer (DUSTiNGS) is an infrared (IR) survey of 50 dwarf galaxies in and around the Local Group designed to detect evolved stars in the dust-producing phase.

Massive dusty evolved stars such as luminous blue variables, Wolf-Rayet stars, red supergiants, and supergiant $\mathrm{B}[\mathrm{e}]$ stars are prolific dust producers (Smith 2014; Bonanos et al. 2010; Kastner et al. 2006; Voors et al. 2000; Smith et al. 2003), though it is uncertain how much, if any, dust will survive the subsequent supernova ( $\mathrm{SN}$ ) explosion. The role of episodic mass loss, which is often accompanied by dust production, in the evolution of massive stars remains an open question. The inferred presence of pre-existing circumstellar material around several core-collapse SNe (Smith et al. 2007) suggests that mass loss plays an important part in stellar evolution. The DUSTiNGS survey includes a large sample of nearby dwarf galaxies to increase the known sample of these short-lived stars over a wide range of stellar masses and metallicities.

Intermediate-mass asymptotic giant branch (AGB) stars condense dust from material formed in situ and may be a major source of interstellar dust (Gehrz 1989) as inferred, for example, by the AGB origin of a large fraction of presolar grains found in meteorites (e.g., Gail et al. 2009). Several works have shown that a small population of very dusty AGB stars dominate the AGB dust production in the Magellanic Clouds at a given time (Srinivasan et al. 2009; Boyer et al. 2012; Riebel et al. 2012; Zhukovska \& Henning 2013; Schneider et al. 2014). These stars (sometimes called "extreme" AGB stars-or x-AGB stars) are optically obscured, and are generally selected via their red colors ([3.6]-[8] > 3 mag; see Section 6.1.2). They comprise $\lesssim 5 \%$ of the AGB population, but produce more than three-fourths of the AGB dust. Through spectral energy distribution modeling, Riebel et al. (2012) find that most of these stars in the Large Magellanic Cloud are carbon rich. Carbon stars form easily in 
metal-poor environments because of a low initial oxygen abundance in the circumstellar envelope and hence more free carbon after the formation of C/O (e.g., Groenewegen \& de Jong 1993). It follows that carbon stars may also dominate the dust production in the more metal-poor dwarf galaxies.

Nevertheless, it is still unclear how much AGB dust survives the harsh environment of the ISM produced by SN shocks (e.g., Jones \& Nuth 2011). Recent Spitzer observations of the Small and Large Magellanic Clouds (SMC/LMC) by the Surveying the Agents of Galaxy Evolution (SAGE) program (Meixner et al. 2006; Gordon et al. 2011) produced a complete census of AGB stars in those galaxies. Estimates of the total dust input compared with other known dust sources (i.e., SNe ejecta) indicate that AGB stars may be the dominant source of stellarproduced dust grains (Matsuura et al. 2009; Boyer et al. 2012; Riebel et al. 2012; Zhukovska \& Henning 2013; Schneider et al. 2014). These works also concluded that, despite their efficient dust production, AGB stars can account for only a fraction of the ISM dust mass in the SMC and LMC. However, a revised measurement of the SMC and LMC ISM dust masses using Herschel Space Observatory data is significantly smaller than previously estimated with Spitzer data (Gordon 2014), indicating that AGB stars may in fact be a dominant dust source in these galaxies.

\subsection{The Metallicity Dependence of Dust Production}

For more metal-poor populations, the metallicity dependence of dust production by AGB stars remains unclear. Some AGB stellar evolution models suggest that dust production easily occurs at very low metallicity because carbon stars create carbon in situ (e.g., Karakas \& Lattanzio 2007; Mattsson et al. 2008; Wachter et al. 2008). Other models suggest that at very low metallicity $([\mathrm{Fe} / \mathrm{H}] \lesssim-2)$, AGB stars contribute little dust and thus provide a negligible contribution to the total dust budget of high-redshift galaxies (L. Mattsson, in preparation).

The effect of the metallicity on dust production likely differs for oxygen-rich and carbon-rich AGB stars because carbon stars create their own carbon. Photometric surveys of metal-poor globular clusters show modest dust production by low-mass oxygen-rich AGB stars (Boyer et al. 2009a; McDonald et al. $2011 \mathrm{a}, 2011 \mathrm{~b}$ ) down to $[\mathrm{Fe} / \mathrm{H}] \approx-1.7$. Infrared spectroscopy of O-rich AGB stars in the Magellanic Clouds and globular clusters reveals trends consistent with reduced dust production at lower metallicities, as expected due to reduced amounts of the oxygen needed to make silicate dust, but these studies are not conclusive (Sloan et al. 2008, 2010; Groenewegen et al. 2009).

In carbon stars, some works suggest there is no metallicity dependence on dust formation (e.g., Groenewegen et al. 2007), while some do find hints of such a dependence at $[\mathrm{Fe} / \mathrm{H}] \lesssim-1$ (van Loon et al. 2008; Sloan et al. 2012). However, this latter group includes only two C stars in the Sculptor dwarf and three in Leo I $([\mathrm{Fe} / \mathrm{H}]=-1.68$ and -1.43 , respectively; McConnachie 2012). Larger samples at low metallicities are clearly needed.

The DUSTiNGS survey aims to build statistics of the shortlived dust-producing phase at low metallicity for constraining stellar evolution and dust production models. Here, we present an overview of the survey, which greatly extends the baseline in age and metallicity over previous observations (Table 1, Figure 1), and provides a near-complete census of galaxies within $1.5 \mathrm{Mpc}$ at 3.6 and $4.5 \mu \mathrm{m}$. The purpose of this overview is to describe the DUSTiNGS targets (Section 2), the observations and survey design (Section 3), and the data products
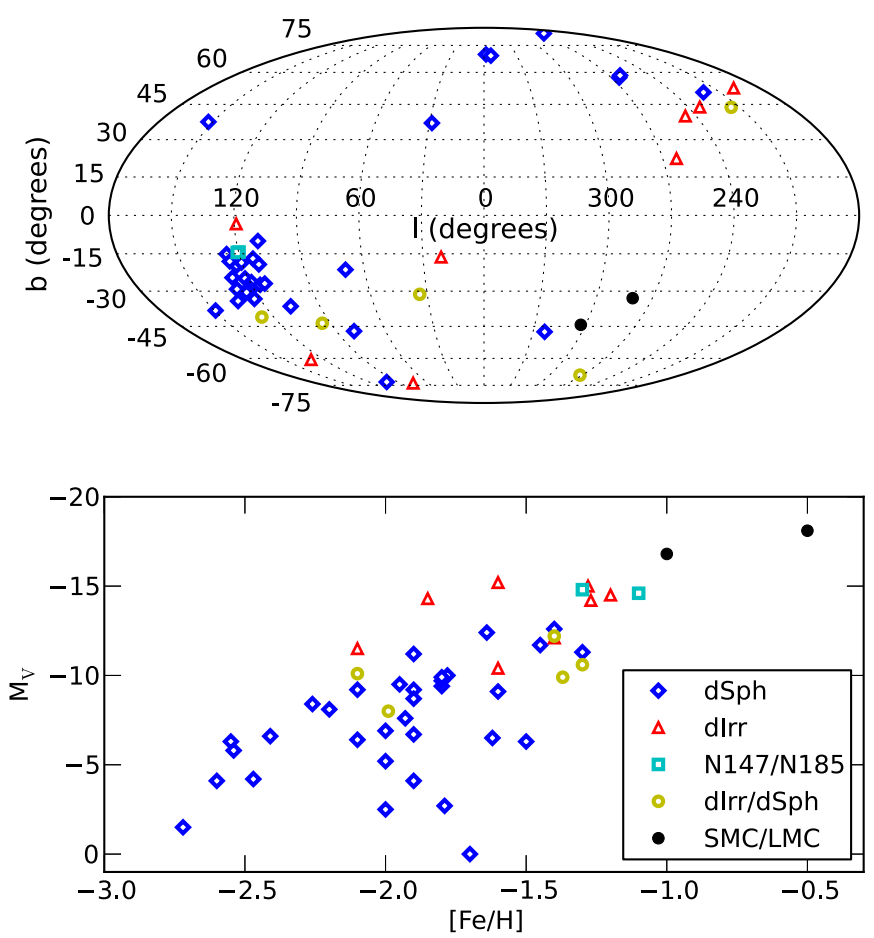

Figure 1. DUSTiNGS targets with properties from Table 1. Upper panel: distribution of target galaxies in Galactic coordinates. Note there are few targets near the Galactic plane/bulge, limiting the effects of foreground extinction and contamination (Sections 5.2 and 5.3). The cluster of dSph galaxies near $l=120^{\circ}$ and $b=-20^{\circ}$ is the Andromeda group. Lower panel: distribution of target galaxies in absolute $V$-band magnitude $\left(M_{\mathrm{V}}\right)$ and metallicity $([\mathrm{Fe} / \mathrm{H}])$. The Small and Large Magellanic Clouds (SMC/LMC) are shown for comparison.

(Sections 4 and 5). We also estimate the AGB population size (Section 6). Forthcoming papers will describe additional scientific results in detail; in Boyer et al. (2014, hereafter Paper II), we identify individual $\mathrm{x}-\mathrm{AGB}$ star candidates via their pulsation.

\section{THE TARGETS}

\subsection{Nearby Dwarf Galaxies}

We describe the DUSTiNGS targets and their properties in Table 1 and Figure 1. Dwarf galaxies are the most prevalent morphological type of galaxy and may be the building blocks of larger galactic systems (Tosi 2003). Additionally, nearby dwarfs present a complete suite of galactic environments (e.g., metallicity and star formation history; Mateo 1998; McConnachie 2012) that is perfect for studying the connection between stellar populations and galaxy evolution. DUSTiNGS includes all dwarf galaxies within $1.5 \mathrm{Mpc}$ that were known at the time of the observations and that lacked sufficient coverage with Spitzer (see below). The next nearest galaxy $(d=1.7 \mathrm{Mpc})$ is beyond Spitzer's ability to resolve stars. Following McConnachie (2012), we divide the nearby resolved dwarfs into dwarf spheroidals (dSphs), dwarf irregulars (dIrrs), and transition (dIrr/dSph, or dTrans) galaxies.

The dSphs typically have no detected neutral hydrogen and show no evidence of recent star formation (within the last $200 \mathrm{Myr}$ ). The dSph galaxies are thought to have had their star formation terminated either through an internal process such as a galactic wind (e.g., Dekel \& Silk 1986), an external process such as an interaction with a more massive host galaxy (e.g., Mayer et al. 2001, 2006), or heating by the ultraviolet field associated with reionization (e.g., Babul \& Rees 1992; Efstathiou 1992). 
Table 1

Adopted Target Parameters

\begin{tabular}{|c|c|c|c|c|c|c|c|c|}
\hline Galaxy & $\begin{array}{c}\text { R.A. } \\
(\mathrm{J} 2000)\end{array}$ & $\begin{array}{c}\text { Decl. } \\
(\mathrm{J} 2000)\end{array}$ & $\begin{array}{c}(m-M)_{0} \\
(\mathrm{mag})\end{array}$ & $\begin{array}{c}M_{\mathrm{V}} \\
(\mathrm{mag})\end{array}$ & $12+\log (\mathrm{O} / \mathrm{H})$ & {$[\mathrm{Fe} / \mathrm{H}]$} & $\begin{array}{l}r_{\mathrm{h}} \\
\left(^{\prime}\right)\end{array}$ & References \\
\hline \multicolumn{9}{|c|}{ Dwarf spheroidals (dSph) } \\
\hline And XVIII & 000214.5 & +450520 & $25.66 \pm 0.13$ & $-9.7 \pm 0.1$ & $\ldots$ & $-1.80 \pm 0.10$ & $0.92 \pm 0.06$ & 1 \\
\hline And $\mathrm{XX}$ & 000730.7 & +350756 & $24.35_{-0.15}^{+0.12}$ & $-6.3_{-0.8}^{+1.1}$ & $\cdots$ & $-1.50 \pm 0.10$ & $0.53 \pm 0.14$ & 1,2 \\
\hline And XIX & 001932.1 & +350237 & $24.57_{-0.36}^{+0.08}$ & $-9.2 \pm 0.6$ & $\ldots$ & $-1.90 \pm 0.10$ & $6.20 \pm 0.10$ & 1,2 \\
\hline Cetus & 002611.0 & -110240 & $24.39 \pm 0.07$ & $-11.2 \pm 0.2$ & $\ldots$ & $-1.90 \pm 0.10$ & $3.20 \pm 0.10$ & 1 \\
\hline NGC 147 & 003312.1 & +483032 & $24.15 \pm 0.09$ & $-14.6 \pm 0.1$ & $\cdots$ & $-1.10 \pm 0.10$ & 3.17 & 1 \\
\hline And III & 003533.8 & +362952 & $24.37 \pm 0.07$ & $-10.0 \pm 0.3$ & $\ldots$ & $-1.78 \pm 0.04$ & $2.20 \pm 0.20$ & 1 \\
\hline And XVII & 003707.0 & +441920 & $24.50 \pm 0.10$ & $-8.7 \pm 0.4$ & $\ldots$ & $-1.90 \pm 0.20$ & $1.24 \pm 0.08$ & 1 \\
\hline NGC 185 & 003858.0 & +482015 & $23.95 \pm 0.09$ & $-14.8 \pm 0.1$ & $8.20 \pm 0.20$ & $-1.30 \pm 0.10$ & 2.55 & 1,3 \\
\hline And I & 004539.8 & +380228 & $24.36 \pm 0.07$ & $-11.7 \pm 0.1$ & $\ldots$ & $-1.45 \pm 0.04$ & $3.10 \pm 0.30$ & 1 \\
\hline And XI & 004620.0 & +334805 & $24.40_{-0.50}^{+0.20}$ & $-6.9 \pm 1.3$ & $\cdots$ & $-2.00 \pm 0.20$ & $0.71 \pm 0.03$ & 1 \\
\hline And XII & 004727.0 & +342229 & $24.70 \pm 0.30$ & $-6.4 \pm 1.2$ & $\ldots$ & $-2.10 \pm 0.20$ & $1.20 \pm 0.20$ & 1 \\
\hline And XIV & 005135.0 & +294149 & $24.33 \pm 0.33$ & $-8.4 \pm 0.6$ & $\ldots$ & $-2.26 \pm 0.05$ & $1.70 \pm 0.80$ & 1 \\
\hline And XIII & 005151.0 & +330016 & $24.40_{-0.40}^{+0.33}$ & $-6.7 \pm 1.3$ & $\cdots$ & $-1.90 \pm 0.20$ & $0.78 \pm 0.08$ & 1,2 \\
\hline And IX & 005253.0 & +431145 & $23.89_{-0.08}^{+0.31}$ & $-8.1 \pm 1.1$ & $\ldots$ & $-2.20 \pm 0.20$ & $2.50 \pm 0.10$ & 1,2 \\
\hline And XVI & 005929.8 & +322236 & $23.60 \pm 0.20$ & $-9.2 \pm 0.4$ & $\ldots$ & $-2.10 \pm 0.20$ & $0.89 \pm 0.05$ & 1 \\
\hline And X & 010633.7 & +444816 & $24.23 \pm 0.21$ & $-7.6 \pm 1.0$ & $\ldots$ & $-1.93 \pm 0.11$ & $1.30 \pm 0.10$ & 1 \\
\hline And V & 011017.1 & +473741 & $24.44 \pm 0.08$ & $-9.1 \pm 0.2$ & $\ldots$ & $-1.60 \pm 0.30$ & $1.40 \pm 0.20$ & 1 \\
\hline And XV & 011418.7 & +380703 & $24.00 \pm 0.20$ & $-9.4 \pm 0.4$ & $\ldots$ & $-1.80 \pm 0.20$ & $1.21 \pm 0.05$ & 1 \\
\hline And II & 011629.8 & +332509 & $24.07 \pm 0.06$ & $-12.4 \pm 0.2$ & $\ldots$ & $-1.64 \pm 0.04$ & $6.20 \pm 0.20$ & 1 \\
\hline And XXII & 012740.0 & +280525 & $24.82_{-0.31}^{+0.07}$ & $-6.5 \pm 0.8$ & $\ldots$ & $-1.62 \pm 0.05$ & $0.94 \pm 0.10$ & $1,4,5$ \\
\hline Segue 2 & 021916.0 & +201031 & $17.70 \pm 0.10$ & $-2.5 \pm 0.3$ & $\ldots$ & $-2.00 \pm 0.25$ & $3.40 \pm 0.20$ & 1 \\
\hline UMa II & 085130.0 & +630748 & $17.50 \pm 0.30$ & $-4.2 \pm 0.6$ & $\ldots$ & $-2.47 \pm 0.06$ & $16.0 \pm 1.0$ & 1 \\
\hline Segue 1 & 100704.0 & +160455 & $16.80 \pm 0.20$ & $-1.5 \pm 0.8$ & $\ldots$ & $-2.72 \pm 0.40$ & $4.4_{-0.6}^{+1.2}$ & 1 \\
\hline Willman 1 & 104921.0 & +510300 & $17.90 \pm 0.40$ & $-2.7 \pm 0.8$ & $\ldots$ & -2.10 & $2.30 \pm 0.40$ & 1 \\
\hline Leo V & 113109.6 & +021312 & $21.25 \pm 0.12$ & $-5.2 \pm 0.4$ & $\ldots$ & $-2.00 \pm 0.20$ & $2.60 \pm 0.60$ & 1 \\
\hline Leo IV & 113257.0 & -003200 & $20.94 \pm 0.09$ & $-5.8 \pm 0.4$ & $\ldots$ & $-2.54 \pm 0.07$ & $4.60 \pm 0.80$ & 1 \\
\hline Coma Beren & 122659.0 & +235415 & $18.20 \pm 0.20$ & $-4.1 \pm 0.5$ & $\ldots$ & $-2.60 \pm 0.05$ & $6.00 \pm 0.60$ & 1 \\
\hline CVn II & 125710.0 & +341915 & $21.02 \pm 0.06$ & $-4.9 \pm 0.5$ & $\ldots$ & $-2.20 \pm 0.05$ & $1.60 \pm 0.30$ & 1 \\
\hline Bootes II & 135800.0 & +125100 & $18.10 \pm 0.06$ & $-2.7 \pm 0.9$ & $\ldots$ & $-1.79 \pm 0.05$ & $4.20 \pm 1.40$ & 1 \\
\hline Bootes I & 140006.0 & +143000 & $19.11 \pm 0.08$ & $-6.3 \pm 0.2$ & $\ldots$ & $-2.55 \pm 0.11$ & $12.6 \pm 1.0$ & 1 \\
\hline Hercules DW & 163102.0 & +124730 & $20.60 \pm 0.20$ & $-6.6 \pm 0.4$ & $\ldots$ & $-2.41 \pm 0.04$ & $8.6_{-1.1}^{+1.8}$ & 1 \\
\hline Segue $3^{a}$ & 212131.1 & +190703 & $16.1 \pm 0.1$ & $-0.0 \pm 0.8$ & $\ldots$ & $-1.7_{-0.3}^{+0.1}$ & $0.47 \pm 0.13$ & 6 \\
\hline Tucana & 224149.6 & -642510 & $24.74 \pm 0.12$ & $-9.5 \pm 0.2$ & $\ldots$ & $-1.95 \pm 0.15$ & $1.10 \pm 0.20$ & 1 \\
\hline Pisces II & 225831.0 & +055709 & $21.31 \pm 0.18$ & $-4.1 \pm 0.4$ & $\ldots$ & -1.90 & $1.10 \pm 0.10$ & 1,7 \\
\hline And VII & 232631.7 & +504033 & $24.41 \pm 0.10$ & $-12.6 \pm 0.3$ & $\ldots$ & $-1.40 \pm 0.30$ & $3.50 \pm 0.10$ & 1 \\
\hline And VI & 235146.3 & +243457 & $24.47 \pm 0.07$ & $-11.3 \pm 0.2$ & $\ldots$ & $-1.30 \pm 0.14$ & $2.30 \pm 0.20$ & 1 \\
\hline And XXI & 235447.7 & +422815 & $24.67 \pm 0.13$ & $-9.9 \pm 0.6$ & $\cdots$ & $-1.80 \pm 0.20$ & $3.50 \pm 0.30$ & 1 \\
\hline \multicolumn{9}{|c|}{ Dwarf irregulars (dIrr) } \\
\hline WLM & 000158.2 & -152739 & $24.95 \pm 0.03$ & $-14.2 \pm 0.1$ & $7.83 \pm 0.06$ & $-1.27 \pm 0.04$ & 7.78 & $1,8,9$ \\
\hline IC 10 & 002017.3 & +591814 & $24.27 \pm 0.18$ & $-15.0 \pm 0.2$ & $8.19 \pm 0.15$ & -1.28 & 2.65 & $1,3,10$ \\
\hline IC 1613 & 010447.8 & +020704 & $24.39 \pm 0.12$ & $-15.2 \pm 0.2$ & $7.62 \pm 0.05$ & $-1.60 \pm 0.20$ & 6.81 & $1,8,11$ \\
\hline Leo A & 095926.5 & +304447 & $24.51 \pm 0.12$ & $-12.1 \pm 0.2$ & $7.35 \pm 0.06$ & $-1.40 \pm 0.20$ & 2.15 & $1,8,9,12$ \\
\hline Sextans B & 100000.1 & +051956 & $25.60 \pm 0.03$ & $-14.5 \pm 0.2$ & $7.53 \pm 0.05$ & -1.6 & $1.06 \pm 0.10$ & $1,3,8,9$ \\
\hline Antlia & 100404.1 & -271952 & $25.65 \pm 0.10$ & $-10.4 \pm 0.2$ & $\ldots$ & $-1.60 \pm 0.10$ & $1.20 \pm 0.12$ & 1,13 \\
\hline Sextans A & 101100.8 & -044134 & $25.60 \pm 0.03$ & $-14.3 \pm 0.1$ & $7.54 \pm 0.06$ & -1.85 & 2.47 & $1,8,9$ \\
\hline Sag DIG & 192959.0 & -174041 & $25.35 \pm 0.18$ & $-11.5 \pm 0.3$ & $7.42 \pm 0.30$ & $-2.10 \pm 0.20$ & $0.91 \pm 0.05$ & $1,3,13$ \\
\hline \multicolumn{9}{|c|}{ Transition dwarfs (dTrans or dIrr/dSph) } \\
\hline LGS 3 & 010355.0 & +215306 & $23.96_{-0.07}^{+0.10}$ & $-10.1 \pm 0.1$ & $\cdots$ & $-2.10 \pm 0.22$ & $2.10 \pm 0.20$ & 1,14 \\
\hline Phoenix & 015106.3 & -442641 & $23.09 \pm 0.10$ & $-9.9 \pm 0.4$ & $\ldots$ & $-1.37 \pm 0.20$ & 3.76 & 1,15 \\
\hline Leo $\mathrm{T}$ & 093453.4 & +170305 & $23.10 \pm 0.10$ & $-8.0 \pm 0.5$ & $\ldots$ & $-1.99 \pm 0.05$ & $0.99 \pm 0.06$ & 1 \\
\hline Aquarius & 204651.8 & -125053 & $25.15 \pm 0.08$ & $-10.6 \pm 0.1$ & $\ldots$ & $-1.30 \pm 0.20$ & $1.47 \pm 0.04$ & 1,16 \\
\hline Pegasus & 232836.3 & +144435 & $24.82 \pm 0.07$ & $-12.2 \pm 0.2$ & $7.93 \pm 0.13$ & $-1.40 \pm 0.20$ & 2.10 & $1,8,9,16$ \\
\hline
\end{tabular}

Notes. The half-light radius $\left(r_{\mathrm{h}}\right)$ is the distance along the semimajor axis that contains half the visible light of the galaxy.

${ }^{a}$ Segue 3 is likely a stellar cluster (e.g., Belokurov et al. 2010).

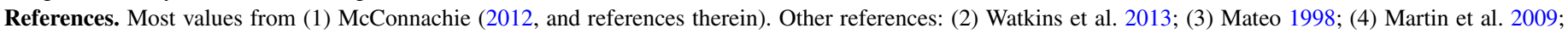

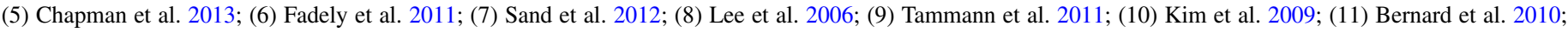
(12) Bellazzini et al. 2014; (13) Pimbblet \& Couch 2012; (14) Miller et al. 2001; (15) Menzies et al. 2008; and (16) McConnachie et al. 2005. 
The dIrrs are gas rich and show evidence of $\mathrm{H}$ II regions that are sites of current massive star formation. The dTrans galaxies are typically gas rich, but show no evidence of current massive star formation through the presence of $\mathrm{H}$ II regions. The nature of transition galaxies is a matter of debate. Many dTrans galaxies are consistent with dIrr galaxies that are forming stars at such a low rate that the absence of $\mathrm{H}$ II regions is consistent with stochastic variations. However, some show evidence for reduced gas mass fractions and apparently lie between the dSphs and dIrrs in the morphology-density relationship (e.g., Skillman et al. 2003; Weisz et al. 2011).

Most of the DUSTiNGS galaxies are members of the Local Group (Mateo 1998; McConnachie 2012). Based on their heliocentric radial velocities, van den Bergh (1999) argues that Sextans A, Sextans B, and Antlia are not Local Group members, but instead belong to a subgroup with NGC 3109 that is expanding with the Hubble flow (van den Bergh 1999).

Of those known before our observations, we exclude 15 galaxies within 1.5 Mpc from DUSTiNGS because of existing Spitzer observations. Nine of the most nearby dSph galaxies were observed in cycle 5 using a similar observing strategy to the one employed here (PI: P. Barmby, PID 50134: CVn I, Draco, Fornax, Leo I, Leo II, Sculptor, Sextans, UMi, and UMa I). Carina, NGC 3109, NGC 6822, and NGC 205 were also covered by several Spitzer programs (PIDs: 128, 159, 3126, 3400, 20469, 40204, 61001, 70062). CMa and Sgr dSph are too large on the sky for efficient Spitzer imaging. Because they are also nearby (7 and $26 \mathrm{kpc}$, respectively), the Wide-Field Survey Explorer (WISE; Wright et al. 2010) all-sky IR survey is sufficiently sensitive to detect a large fraction of the dust-producing stars.

\subsection{Expected Dusty Stellar Populations}

Galaxies with different morphological types are expected to host different sized AGB and massive star populations based on both the typical mass scales and recent star formation histories. For example, dIrrs are typically more massive than the dSphs in the Local Group, so they should have a larger population of dusty stars. However, the level of recent star formation activity plays a significant role in determining the number of these stars per unit stellar mass of a galaxy. An intermediate-mass star enters the AGB stage of stellar evolution between about $100 \mathrm{Myr}$ and 3 Gyr after formation depending on its initial mass (Marigo et al. 2013). Thus, galaxies with higher rates of star formation over these timescales will have larger populations of AGB stars and galaxies with more recent star formation will have massive stars. Because of the higher gas-rich content of dIrrs relative to dSphs, the two factors of stellar mass and recent star formation activity often compound one another.

However, differences do exist within each morphological type, with dSphs showing the greatest divergence in recent star formation activity (Weisz et al. 2014), adding some uncertainty to expectations on the AGB population from this morphological type. Detailed studies of individual galaxies have shown that delayed onset of star formation is also possible. Both Leo A (Cole et al. 2007) and Leo T (Weisz et al. 2012) are examples of gas-rich galaxies that have formed the majority of their stars within the last 5-8 Gyr. Based on their overall lower mass, the number of AGB stars in each of these systems may be low even though a significant fraction of stellar mass in each galaxy was formed over the timescale of interest.

\section{SURVEY DESIGN}

The DUSTiNGS survey includes uniform 3.6 and $4.5 \mu \mathrm{m}$ imaging of 50 nearby galaxies. These filters are particularly suited for identifying sources with warm dust (e.g., see the spectral energy distributions of dusty stars in Figure 26 from Boyer et al. 2011). The observations are summarized in Table 2. DUSTiNGS uses the InfraRed Array Camera (IRAC; Fazio et al. 2004) on board the Spitzer Space Telescope (Werner et al. 2004; Gehrz et al. 2007) during the post-cryogen phase. The spatial coverage extends to beyond the half-light radius $\left(r_{\mathrm{h}}\right.$; or the distance along the semimajor axis that contains half the visible light of the galaxy) at each wavelength for determining the level of foreground and background contaminating point sources. Each galaxy was observed at two epochs approximately six months apart to provide an additional diagnostic for identifying AGB stars, which are variable at these wavelengths (e.g., Le Bertre 1992, 1993; McQuinn et al. 2007; Vijh et al. 2009). The imaging footprint for Wolf-Lundmark-Mellote (WLM) is shown in Figure 2 as an example of the DUSTiNGS mapping scheme.

Stellar evolution models (e.g., Bressan et al. 2012; Marigo et al. 2013) and previous studies at these wavelengths (e.g., Jackson et al. 2007a, 2007b; Boyer et al. 2009b) show that the tip of the red giant branch (TRGB) is located at absolute $3.6 \mu \mathrm{m}$ magnitude $-6.6 \lesssim M_{3.6} \lesssim-6 \mathrm{mag}$. Thus, to ensure that the majority of thermally pulsing (TP) AGB stars and dust-producing massive stars would be detected, the exposure times were chosen so that the $3 \sigma$ detection limit is at least one magnitude fainter than $M_{3.6}=-6 \mathrm{mag}$. Together, the extended areal coverage and sensitivity enable the detection of most of the evolved stellar populations, thus significantly improving the statistics on these short-lived evolutionary phases. In particular, the DUSTiNGS sensitivity limit ensures the detection of nearly all of the X-AGB stars; in the Magellanic Clouds, $>96 \%$ of the X-AGB stars are brighter than $M_{3.6}=-8$ mag (Section 6.1.2).

For galaxies more distant than $400 \mathrm{kpc}$, we obtained 36 dithered frames with $30 \mathrm{~s}$ exposures at each map position (deep observations, $m_{3.6}^{5 \sigma} \approx 20.5 \mathrm{mag}$ ), one half of these frames were obtained in each epoch. Similarly, for galaxies with $130<d<$ $400 \mathrm{kpc}$, we obtained 5 dithered frames with $30 \mathrm{~s}$ exposures (medium, $m_{3.6}^{5 \sigma} \approx 19.5 \mathrm{mag}$ ) and for galaxies within $130 \mathrm{kpc}$, we obtained five dithered frames with $12 \mathrm{~s}$ exposures (shallow, $m_{3.6}^{5 \sigma} \approx 18.5 \mathrm{mag}$ ). In each case, we used the small cycling IRAC dither pattern with a median separation of 10.5 pixels to help eliminate imaging artifacts (the IRAC pixel size is $1^{\prime \prime} .22$ ). The map sizes and total exposure times $\left(t_{\exp }\right)$ are listed in Table 2. The co-added, subsampled mosaics are available for download at the Mikulski Archive for Space Telescopes (MAST) ${ }^{14}$ and the InfraRed Science Archive (IRSA), ${ }^{15}$ and we show examples in Figure 13.

\section{POINT-SOURCE PHOTOMETRY}

We describe below the photometry for the DUSTiNGS survey, including the photometric corrections, saturation, completeness, and crowding. The final photometric catalogs are available via MAST, ${ }^{14}$ IRSA, ${ }^{15}$ and VizieR. ${ }^{16}$

\footnotetext{
14 https://archive.stsci.edu/

15 http://irsa.ipac.caltech.edu/frontpage/

16 http://vizier.u-strasbg.fr/viz-bin/VizieR
} 
Table 2

Data and Observations

\begin{tabular}{|c|c|c|c|c|c|c|c|c|c|c|}
\hline Galaxy & $\begin{array}{c}\left\langle t_{\text {exp }}\right\rangle^{\mathrm{a}} \\
(\mathrm{s})\end{array}$ & $\begin{array}{l}5 \sigma^{\mathrm{a}} \\
(\mu \mathrm{Jy})\end{array}$ & $\begin{array}{l}\text { Map }^{b} \\
\text { Size }\end{array}$ & $\mathrm{AOR}^{\mathrm{e}} \mathrm{Key}$ & $\begin{array}{l}\text { Obs. Date } \\
\text { (UTC) }\end{array}$ & AOR Key & $\begin{array}{l}\text { Obs. Date } \\
\text { (UTC) }\end{array}$ & $\begin{array}{c}\text { Separation } \\
\text { (days) }\end{array}$ & $N_{\mathrm{ptsrc}} \mathrm{c}$ & $\begin{array}{l}\text { Coverage }^{\mathrm{d}} \\
\left(\operatorname{arcmin}^{2}\right)\end{array}$ \\
\hline & & & & \multicolumn{2}{|c|}{ Epoch 1} & \multicolumn{2}{|c|}{ Epoch 2} & & & \\
\hline And I & 1080 & 1.6 & $2 \times 3$ & 42307328 & 2011 Sep 8 & 42307584 & 2012 Mar 19 & 193.1 & 4640 & 85.6 \\
\hline And II & 1080 & 1.6 & $2 \times 3$ & 42307840 & 2011 Sep 16 & 42308096 & 2012 Mar 15 & 181.3 & 4309 & 85.4 \\
\hline And III & 1080 & 1.6 & $2 \times 3$ & 42308352 & 2011 Sep 24 & 42308608 & 2012 Mar 26 & 184.7 & 4043 & 85.9 \\
\hline And V & 1080 & 1.6 & $2 \times 3$ & 42309376 & 2011 Sep 21 & 42309632 & 2012 Mar 27 & 188.0 & 4877 & 85.8 \\
\hline And VI & 1080 & 1.6 & $2 \times 3$ & 42309888 & 2011 Sep 24 & 42310144 & 2012 Mar 9 & 167.8 & 4189 & 81.9 \\
\hline And VII & 1080 & 1.6 & $2 \times 3$ & 42310400 & 2011 Aug 29 & 42310656 & $2012 \operatorname{Mar} 20$ & 203.4 & 6951 & 79.9 \\
\hline And IX & 1080 & 1.6 & $2 \times 3$ & 42308864 & 2011 Sep 23 & 42309120 & $2012 \operatorname{Mar} 27$ & 186.3 & 4310 & 86.4 \\
\hline And X & 1080 & 1.6 & $2 \times 3$ & 42310912 & 2011 Sep 19 & 42311168 & 2012 Mar 17 & 180.3 & 4826 & 83.3 \\
\hline And XI & 1080 & 1.6 & $2 \times 3$ & 42311424 & 2011 Sep 8 & 42311680 & 2012 Mar 26 & 200.4 & 3200 & 83.9 \\
\hline And XII & 1080 & 1.6 & $2 \times 3$ & 42311936 & 2011 Sep 24 & 42312192 & 2012 Mar 27 & 185.7 & 3739 & 86.1 \\
\hline And XIII & 1080 & 1.6 & $2 \times 3$ & 42312448 & 2011 Sep 21 & 42312704 & 2012 Mar 27 & 188.4 & 3469 & 86.4 \\
\hline And XIV & 1080 & 1.6 & $2 \times 3$ & 42312960 & 2011 Sep 19 & 42313216 & 2012 Mar 21 & 183.9 & 3211 & 86.0 \\
\hline And XV & 1080 & 1.6 & $2 \times 3$ & 42313984 & 2011 Sep 19 & 42314240 & 2012 Mar 16 & 178.9 & 3794 & 84.5 \\
\hline And XVI & 1080 & 1.6 & $2 \times 3$ & 42314496 & 2011 Sep 16 & 42314752 & 2012 Mar 21 & 187.3 & 3164 & 86.5 \\
\hline And XVII & 1080 & 1.6 & $2 \times 3$ & 42315008 & 2011 Sep 23 & 42315264 & 2012 Mar 27 & 186.1 & 4736 & 86.2 \\
\hline And XVIII & 1080 & 1.6 & $2 \times 3$ & 42315520 & 2011 Sep 6 & 42315776 & 2012 Mar 17 & 193.3 & 4297 & 85.2 \\
\hline And XIX & 1080 & 1.6 & $2 \times 3$ & 42313472 & 2011 Sep 24 & 42313728 & 2012 Mar 17 & 175.6 & 3824 & 83.2 \\
\hline And XX & 1080 & 1.6 & $2 \times 3$ & 42316032 & 2011 Aug 29 & 42316288 & 2012 Mar 18 & 201.7 & 2992 & 82.8 \\
\hline And XXI & 1080 & 1.6 & $2 \times 3$ & 42329856 & 2011 Sep 23 & 42330112 & 2012 Mar 19 & 178.2 & 4505 & 82.8 \\
\hline And XXII & 1080 & 1.6 & $2 \times 3$ & 42330368 & 2011 Sep 16 & 42330624 & 2012 Mar 15 & 181.2 & 3121 & 85.6 \\
\hline Antlia & 1080 & 1.6 & $2 \times 3$ & 42316544 & 2011 Jun 28 & 42316800 & 2012 Feb 3 & 219.6 & 3666 & 76.6 \\
\hline Aquarius & 1080 & 1.6 & $2 \times 3$ & 42319616 & 2011 Jun 22 & 42319872 & 2012 Jan 6 & 197.7 & 3072 & 86.2 \\
\hline Bootes I & 60 & 9.1 & $4 \times 5$ & 42317056 & 2011 Sep 6 & 42317312 & 2012 Mar 13 & 189.2 & 3249 & 354.9 \\
\hline Bootes II & 60 & 9.1 & $2 \times 3$ & 42317568 & 2011 Aug 28 & 42317824 & 2012 Mar 13 & 198.3 & 850 & 79.5 \\
\hline Cetus & 1080 & 1.6 & $2 \times 3$ & 42318592 & 2011 Sep 17 & 42318848 & 2012 Feb 3 & 139.7 & 4041 & 79.8 \\
\hline Coma Beren & 60 & 9.1 & $3 \times 4$ & 42319104 & 2011 Jul 18 & 42319360 & 2012 Mar 13 & 239.7 & 1673 & 168.8 \\
\hline CVn II & 150 & 4.4 & $2 \times 3$ & 42318080 & $2011 \mathrm{Jul} 26$ & 42318336 & 2012 Mar 13 & 231.4 & 2037 & 70.6 \\
\hline Hercules Dw & 150 & 4.4 & $3 \times 4$ & 42320640 & 2011 Sep 20 & 42320896 & 2012 Apr 24 & 216.3 & 5434 & 174.2 \\
\hline IC 10 & 1080 & 1.6 & $3 \times 4$ & 42321152 & 2011 Sep 24 & 42321408 & 2012 Apr 4 & 193.1 & 48057 & 195.9 \\
\hline IC 1613 & 1080 & 1.6 & $4 \times 5$ & 42321664 & 2011 Sep 21 & 42321920 & 2012 Feb 20 & 153.2 & 23538 & 356.3 \\
\hline Leo A & 1080 & 1.6 & $2 \times 3$ & 42322944 & 2012 Jan 9 & 42322688 & 2012 Jun 21 & 164.0 & 3680 & 83.1 \\
\hline Leo IV & 150 & 4.4 & $2 \times 3$ & 42323200 & 2011 Jul 18 & 42323456 & 2012 Feb 15 & 212.4 & 1462 & 79.9 \\
\hline Leo $\mathrm{T}$ & 1080 & 1.6 & $2 \times 3$ & 42323968 & 2012 Jan 8 & 42323712 & 2012 Jun 21 & 165.5 & 3394 & 86.1 \\
\hline Leo V & 150 & 4.4 & $2 \times 3$ & 42331392 & 2011 Jul 17 & 42331648 & 2012 Feb 15 & 213.4 & 1470 & 80.1 \\
\hline LGS 3 & 1080 & 1.6 & $2 \times 3$ & 42322176 & 2011 Sep 21 & 42322432 & $2012 \operatorname{Mar} 19$ & 180.4 & 2558 & 85.9 \\
\hline NGC 147 & 1080 & 1.6 & $3 \times 4$ & 42324224 & 2011 Sep 23 & 42324480 & 2012 Mar 30 & 188.8 & 33748 & 201.3 \\
\hline NGC 185 & 1080 & 1.6 & $3 \times 4$ & 42324736 & 2011 Sep 19 & 42324992 & 2012 Apr 4 & 198.0 & 32021 & 192.5 \\
\hline Pegasus & 1080 & 1.6 & $3 \times 4$ & 42320128 & 2011 Sep 17 & 42320384 & 2012 Jan 23 & 127.5 & 10688 & 179.8 \\
\hline Phoenix & 1080 & 1.6 & $3 \times 4$ & 42325248 & 2011 Sep 9 & 42325504 & 2012 Jan 19 & 131.9 & 9474 & 167.2 \\
\hline Pisces II & 150 & 4.4 & $2 \times 3$ & 42331904 & 2011 Aug 2 & 42332160 & 2012 Jan 12 & 163.6 & 1205 & 77.9 \\
\hline Sag DIG & 1080 & 1.6 & $2 \times 3$ & 42326016 & 2011 Nov 20 & 42325760 & 2012 Jun 10 & 202.3 & 7102 & 85.8 \\
\hline Segue 1 & 60 & 9.1 & $2 \times 3$ & 42326528 & 2012 Feb 1 & 42326272 & 2012 Jun 23 & 142.7 & 718 & 79.7 \\
\hline Segue 2 & 60 & 9.1 & $2 \times 3$ & 42330880 & 2011 Sep 23 & 42331136 & 2012 Mar 15 & 174.1 & 598 & 79.8 \\
\hline Segue 3 & 60 & 9.1 & $2 \times 3$ & 42332416 & 2011 Jul 18 & 42332672 & 2012 Jan 2 & 167.7 & 1048 & 75.1 \\
\hline Sextans A & 1080 & 1.6 & $3 \times 4$ & 42327040 & 2012 Feb 1 & 42326784 & 2012 Jul 19 & 168.9 & 8809 & 196.8 \\
\hline Sextans B & 1080 & 1.6 & $3 \times 4$ & 42327552 & 2012 Feb 1 & 42327296 & 2012 Jun 25 & 145.4 & 9631 & 195.7 \\
\hline Tucana & 1080 & 1.6 & $2 \times 3$ & 42327808 & 2011 Jun 19 & 42328064 & 2011 Nov 12 & 146.1 & 4374 & 72.5 \\
\hline UMa II & 150 & 4.4 & $3 \times 4$ & 42328576 & 2012 Jan 2 & 42328320 & 2012 May 08 & 126.8 & 5056 & 163.1 \\
\hline Willman 1 & 150 & 4.4 & $2 \times 3$ & 42329600 & 2012 Jan 9 & 42329344 & 2012 Jun 5 & 148.4 & 2321 & 70.6 \\
\hline WLM & 1080 & 1.6 & $3 \times 4$ & 42328832 & 2011 Sep 10 & 42329088 & 2012 Feb 1 & 144.0 & 12109 & 185.5 \\
\hline
\end{tabular}

Notes.

a The reported total exposure time per pixel and sensitivity are that of the combined epochs 1 and 2 .

${ }^{b}$ Map size is the number of frames on each axis. A single IRAC frame is $5.2 \times 5 ! 2$.

c Total number of reliable point sources (Section 4) within the spatial coverage listed in the last column.

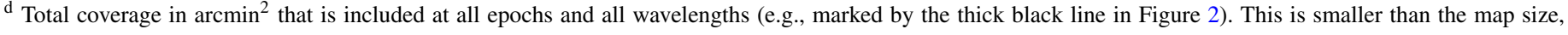

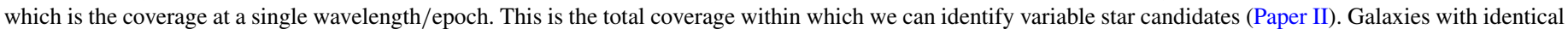
map sizes have slightly different total coverages owing to the rotation between the two epochs.

e Astronomical Observation Request (AOR).

\subsection{PSF Photometry}

Each galaxy in the DUSTiNGS survey was imaged over two epochs. We performed point-spread function (PSF) photometry separately for each epoch to aid in identification of variable stars, and also for a combined epoch to achieve the deepest photometry possible. Stars brighter than $\approx 16 \mathrm{mag}$ (see below) were measured on the individual corrected basic calibrated data 

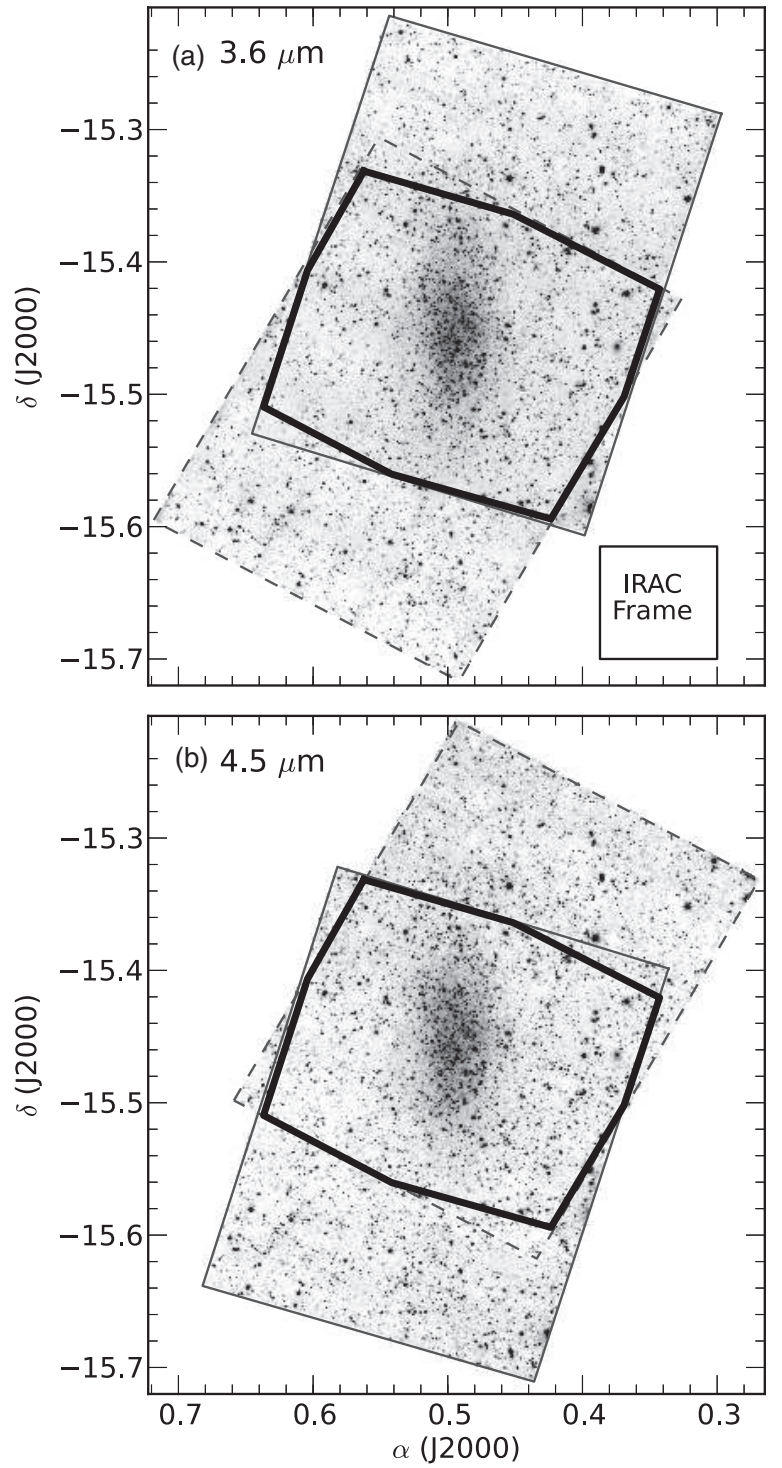

Figure 2. DUSTiNGS mapping strategy. (a) $3.6 \mu \mathrm{m}$ co-added mosaic for Wolf-Lundmark-Mellote (WLM). The solid thin line outlines the epoch 1 coverage and the dashed line outlines the epoch 2 coverage. The thick black line marks the coverage for all wavelengths and epochs, listed in Table 2. (b) Same, for $4.5 \mu \mathrm{m}$. A similar mapping scheme was implemented for every galaxy. For WLM, the coverage is composed of a $3 \times 4$ grid of IRAC frames $(5.2 \times 5.2)$. Table 2 lists the grid size for each galaxy.

(cBCD) frames from the Spitzer processing pipeline versions S18.18.0-S19.1.0 (depending on the date of observations), using a weighted mean to combine the measurements from each frame. The fainter magnitudes were recovered by performing PSF photometry on the co-added cBCD frames, with sub-sampled pixel sizes of 0 .' 6 . This two-step process is necessary to achieve accurate photometry for both the faint and bright sources. The photometry on the individual frames becomes unreliable at faint magnitudes due to the Eddington bias (Eddington 1913). This effect causes stars to appear too bright when approaching the detection limit because the source is more likely to be detected and measured if random fluctuations on the detector make a source brighter than its true flux. On the other hand, bright sources are very sensitive to the details of the PSF (Figure 3), so their fluxes cannot be reliably measured on the mosaic where the PSF features are smeared due to rotation between the frames.
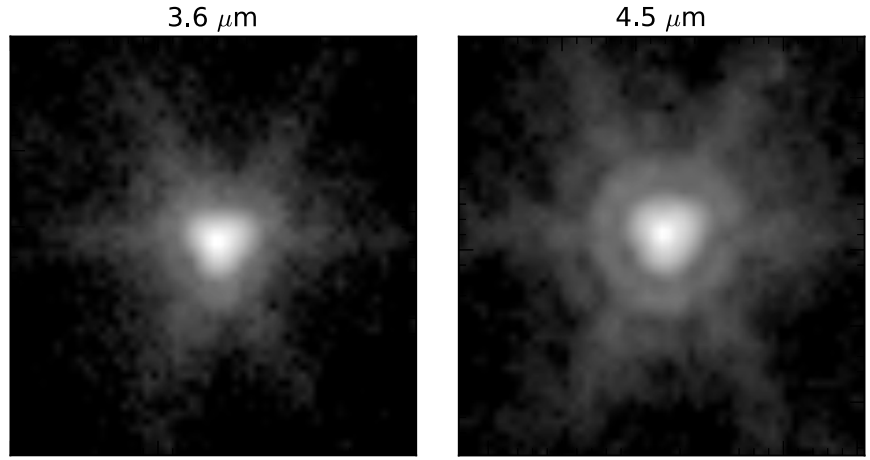

Figure 3. Subsampled ( 0 '. 6 pixels) PSFs, constructed using the data in DAOphot. The images are scaled logarithmically to show the wing structure.

Fainter sources are insensitive to these variations in the PSF and can thus be accurately measured from the mosaic, allowing for the maximum photometric depth.

All PSF photometry was carried out using DAOphot II and ALLSTAR (Stetson 1987), following a similar procedure to that used for the Galactic Plane Survey Extraordinaire (GLIMPSE; Benjamin et al. 2003) and SAGE (Meixner et al. 2006) programs (B. Babler 2013, private communication). The PSF was constructed from the data itself, using the Pegasus dIrr images to select $>10$ bright, isolated stars with well-defined PSF wings (Figure 3). For the photometry on the individual frames, we constructed the PSF using a Moffat function (Moffat 1969) with $\beta=2.5$ for $3.6 \mu \mathrm{m}$ and $\beta=1.5$ for $4.5 \mu \mathrm{m}$ where a larger $\beta$ value approaches a Gaussian. The radius we used to fit the PSF to each source was 1 .' $6-2$ ". 0 , or near the size of the FWHM. For the mosaic photometry, the PSF is different because co-adding the images smears the point sources. To achieve the best match between the cBCD and mosaic photometry, we use a Moffat function $(\beta=1.5)$ and a Lorenz function for the 3.6 and $4.5 \mu \mathrm{m}$ mosaic PSFs, respectively. The fitting radius was set to 3.2 pixels (1".9).

In the final point-source catalog, the transition from cBCD to mosaic photometry occurs at a magnitude where the photometry from both is reliable and agrees to well within the photometric uncertainties. For the medium and deep observations $(150 \mathrm{~s}$ and $1080 \mathrm{~s}$ ), this is at $16.5 \mathrm{mag}$ and $15.7 \mathrm{mag}$ for 3.6 and $4.5 \mu \mathrm{m}$, respectively. For the shallow observations $(60 \mathrm{~s})$, the transition is at $15.5 \mathrm{mag}$ and $15.0 \mathrm{mag}$, respectively. We note that there may be discontinuities in the luminosity functions at the transition point.

\subsection{Photometric Corrections}

We applied several corrections to the photometry, as recommended by the Spitzer Science Center (SSC). First, the cBCD images were corrected for the pixel solid angle variation across the frame (at the level of $1 \%)^{17}$ and converted to data numbers for a robust measure of the photometric uncertainties.

Second, sources were corrected for the variation in the pointsource flux across the array that is a result of the flat-fielding process (the array-location-dependent correction). ${ }^{17}$ This effect can be as high as $10 \%$, depending on the location of the source within the array. This correction is necessary for sources that are on the Rayleigh-Jeans tail within the IRAC filters, which includes most of the sources in our final catalogs. Here, we

\footnotetext{
17 http://irsa.ipac.caltech.edu/data/SPITZER/docs/irac/ iracinstrumenthandbook/home/
} 


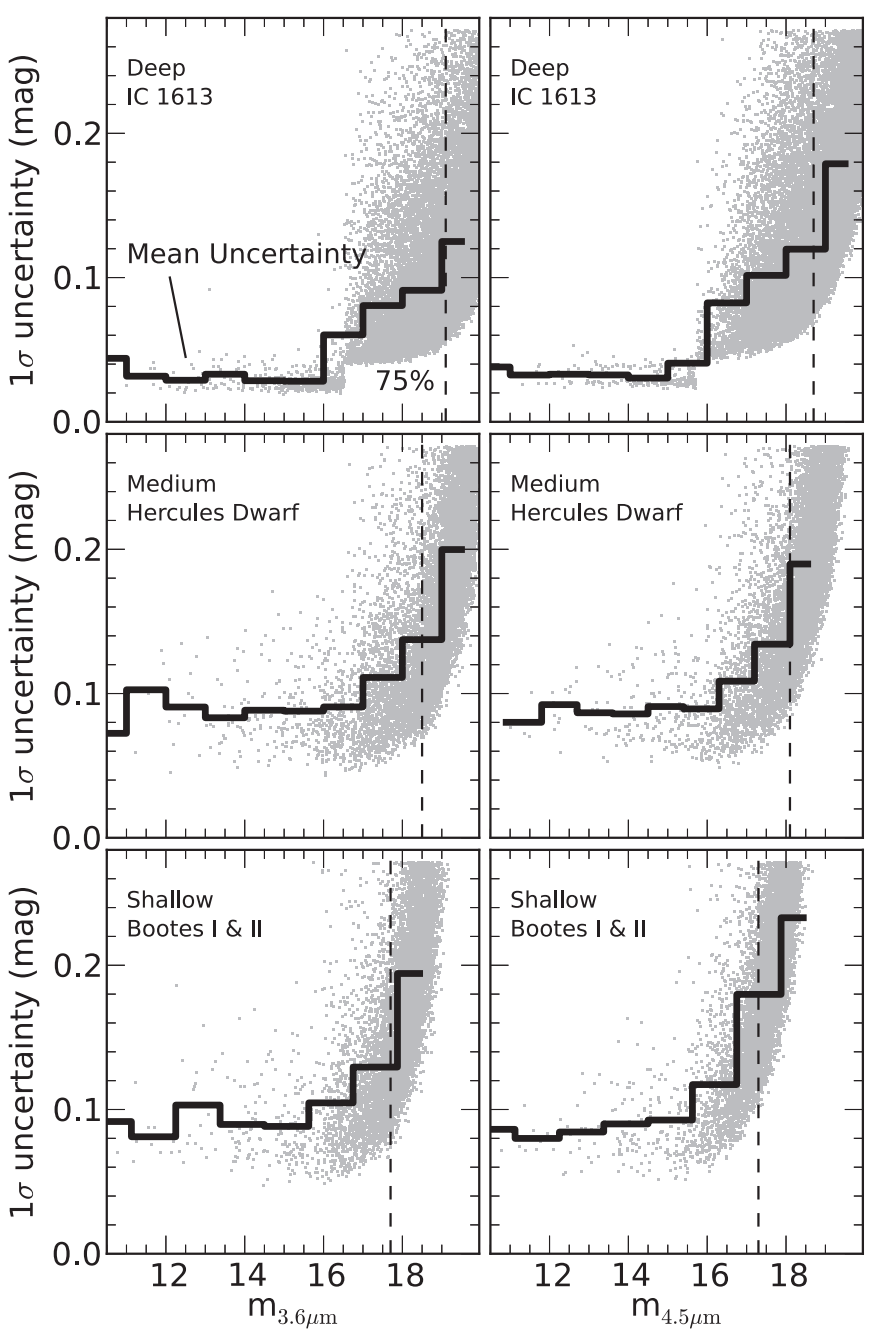

Figure 4. Photometric uncertainties from the Good-Source Catalog (GSC; Section 5) for galaxies observed at all three total exposure times (Table 2): IC 1613 (top), Hercules Dwarf (middle), and both Bootes galaxies (bottom). The Bootes I and II galaxies are combined to illustrate the photometric uncertainties because they both have few point sources. The discontinuities near 16 mag are caused by the use of photometry on the $\mathrm{CBCD}$ frames for brighter sources and on the co-added frames for faint sources. The $75 \%$ completeness level (Section 4.4) is shown as a dashed line in each panel. The histogram indicates the mean uncertainty at a given magnitude.

do not apply the array-location-dependent correction to point sources that show a red color ([3.6]-[4.5] $>0 \mathrm{mag})$ with $\mathrm{a}>3 \sigma$ significance.

Third, fluxes were adjusted by correcting for the location of the center of the point source within a pixel since the quantum efficiency varies across each pixel (the pixel phase correction, up to $4 \%{ }^{17}$ ). Fourth, we applied a color correction for a $3000 \mathrm{~K}$ blackbody to the point-source fluxes, following the SSC's recommendation.

Following Fruchter \& Hook (2002), we increased the measured flux uncertainties by a factor of two to account for correlated uncertainties between the pixels that arise from subsampling the mosaic. This correction was only applied to sources measured from the mosaics. Along with this uncertainty, the final photometric uncertainties include those reported by DAOphot and the calibration uncertainties listed by Reach et al. (2005; Figure 4).

The DUSTiNGS catalog includes magnitudes using the Vegabased zero points of $280.9 \pm 4.1 \mathrm{Jy}$ for $3.6 \mu \mathrm{m}$ and $179.7 \pm 2.6 \mathrm{Jy}$ for $4.5 \mu \mathrm{m} .^{17}$ The final photometry is well matched to that from WISE, which has filters similar to IRAC (3.4 and $4.6 \mu \mathrm{m}$, or W1 and W2). Agreement is within 0.02 mag down to the repeatability limit of the WISE photometry $(\approx 14 \mathrm{mag}) .{ }^{18}$ IRAC point-source positions are accurate to $\approx 0.5 .^{\prime 17}$

\subsection{Saturation}

The saturation limits for $30 \mathrm{~s}$ frames are $10.84 \mathrm{mag}$ and $10.35 \mathrm{mag}$ for 3.6 and $4.5 \mu \mathrm{m}$, respectively. For $12 \mathrm{~s}$ frames, the saturation limits are $9.86 \mathrm{mag}$ and $9.34 \mathrm{mag}$. For the galaxies with $(m-M)_{0}>23 \mathrm{mag}$, saturation only occurs for stars $\gtrsim 2$ mag brighter than the classical AGB limit, which lies near $-10>M_{3.6}>-11 \mathrm{mag}\left(M_{\text {bol }}=-7.1 \mathrm{mag}\right.$, derived for $3.6 \mu \mathrm{m}$ using the models from Groenewegen 2006). This includes all of the dIrr and dTrans galaxies. Because these galaxies are more likely to show evidence of recent/ongoing star formation, they are more likely to include massive AGB stars which approach (and sometimes slightly exceed) the classical AGB limit.

The nearest galaxies with $(m-M)_{0}<22$ mag are all $\mathrm{dSph}$ galaxies with little to no ongoing star formation. Any dust-producing stars in these galaxies are thus more likely to have low initial masses and luminosities near the TRGB. Nevertheless, saturation does occur at magnitudes fainter than the classical AGB limit for nine galaxies (those within the shaded regions in Figures 5(a) and (b): Bootes I, Bootes II, Coma, Hercules, Segue 1, Segue 2, Segue 3, UMa II, and Willman 1.

\subsection{Photometric Completeness}

To assess the repeatability of the photometry, we performed artificial star tests. For each galaxy and wavelength, we added 20 artificial stars of varying magnitudes to a $25 \operatorname{arcmin}^{2}$ region that excludes the galaxy center (crowding in the galaxy centers is discussed in Section 4.5). This was repeated 100 times, for a total of 2000 artificial stars. The magnitude distribution of the fake stars mimicked the real magnitude distribution (see Figure 7). Table 3 lists the mean and the standard deviation in the resulting photometric completeness limits for galaxies unaffected by crowding (Section 4.5) and the completeness curves are shown in Figure 6.

The mean difference between the magnitudes of the added and recovered stars shows a small bias that increases with magnitude, but is $\lesssim 0.06 \mathrm{mag}$ and $0.02 \mathrm{mag}$ for 3.6 and $4.5 \mu \mathrm{m}$, respectively, for stars brighter than the $75 \%$ completeness limit (Figure 7). For stars near 20th magnitude, the mean difference is $\lesssim 0.1 \mathrm{mag}$. This bias is consistent with the effects of point-source crowding, which biases measurements toward brighter magnitudes and increases for faint sources. While only a few galaxies are affected by crowding above the TRGB (Section 4.5), all DUSTiNGS galaxies are affected by crowding at faint magnitudes. The final magnitudes are corrected for this bias.

For most galaxies, the photometry is better than $75 \%$ complete at $M_{3.6}=-6 \mathrm{mag}$ in each epoch, which is the approximate faint limit for the TRGB (Jackson et al. 2007a; Boyer et al. 2009b, 2011). At brighter magnitudes, the completeness rapidly increases (Figure 6); we report the $75 \%$ limit throughout this work because it is representative of the completeness level near the TRGB for the most distant target galaxies. Six DUSTiNGS

\footnotetext{
18 See section VI.3 of the Explanatory Supplement to the WISE All-sky Data Release: http://wise2.ipac.caltech.edu/docs/release/allsky/expsup/index.html.
} 

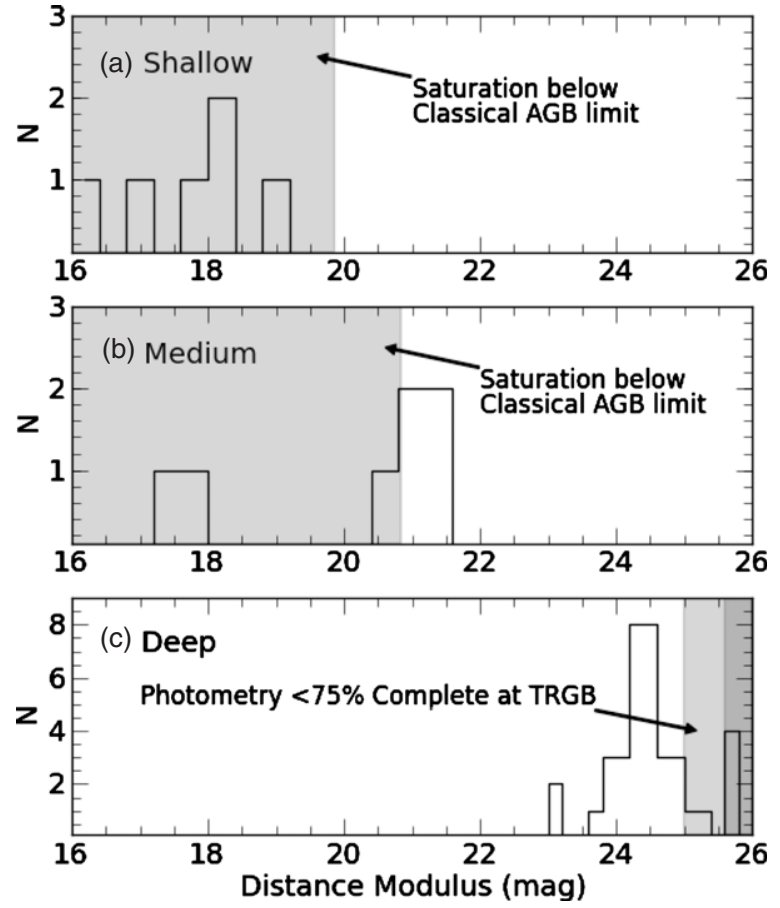

Figure 5. Number distribution of galaxies with saturation and photometric incompleteness for $3.6 \mu \mathrm{m}$. (a) Galaxies with $60 \mathrm{~s}$ total integrations $(12 \mathrm{~s}$ per frame). The shaded region marks the distance moduli where some stars fainter than the classical AGB limit $\left(M_{3.6} \gtrsim-10 \mathrm{mag}\right)$ will saturate. Bright (massive) stars in these galaxies are at risk of saturating. (b) Galaxies with $150 \mathrm{~s}$ total integrations ( $30 \mathrm{~s}$ per frame). Those within the shaded region are at risk of saturating the brightest AGB stars. (c) Galaxies with the deepest (1080 s) integrations ( $30 \mathrm{~s}$ per frame). AGB stars within these galaxies are not at risk of saturation. However, those with distance moduli within the light and dark shaded regions have $<75 \%$ photometric completeness at $M_{3.6}=-6$ mag and $-6.6 \mathrm{mag}$, respectively, which is the assumed range of the TRGB.

Table 3

75\% Photometric Completeness Limits

\begin{tabular}{lcc}
\hline $\begin{array}{l}\left\langle\begin{array}{c}\exp \rangle \\
(\mathrm{s})\end{array}\right. \\
60\end{array}$ & $\begin{array}{c}3.6 \mu \mathrm{m} \\
(\mathrm{mag})\end{array}$ & $\begin{array}{c}4.5 \mu \mathrm{m} \\
(\mathrm{mag})\end{array}$ \\
150 & $17.7 \pm 0.2$ & $17.3 \pm 0.1$ \\
1080 & $18.5 \pm 0.2$ & $18.1 \pm 0.1$ \\
\multicolumn{3}{c}{ Galaxies affected by extrinsic crowding $^{\mathrm{a}}$} \\
\hline And VII & $19.1 \pm 0.1$ & $18.7 \pm 0.2$ \\
IC 10 & $18.5 \pm 0.2$ & $18.5 \pm 0.2$ \\
NGC 147 & $17.7 \pm 0.2$ & $17.7 \pm 0.2$ \\
NGC 185 & $18.2 \pm 0.2$ & $18.3 \pm 0.2$ \\
Sag DIG & $18.6 \pm 0.2$ & $18.4 \pm 0.2$ \\
\hline
\end{tabular}

Notes. Completeness limits $\left(m_{75 \%}\right)$ were computed for the epoch 1 data. For the deeper, combined-epoch photometry, the completeness limit is approximately $0.5 \mathrm{mag}$ fainter. The first three rows list the mean and standard deviation of the completeness limit for galaxies unaffected by crowding. All limits in this table were derived from a $25 \mathrm{arcmin}^{2}$ region away from the galaxy's center.

a All galaxies affected by extrinsic crowding have $\left\langle t_{\exp }\right\rangle=$ $1080 \mathrm{~s}$.

galaxies have $<75 \%$ complete photometry at -6 mag (light shaded region of Figure 5(c)) in a single epoch, though all six reach $75 \%$ completeness by $-6.7 \mathrm{mag}$. In the photometry from the combined epochs, the completeness limit is approximately
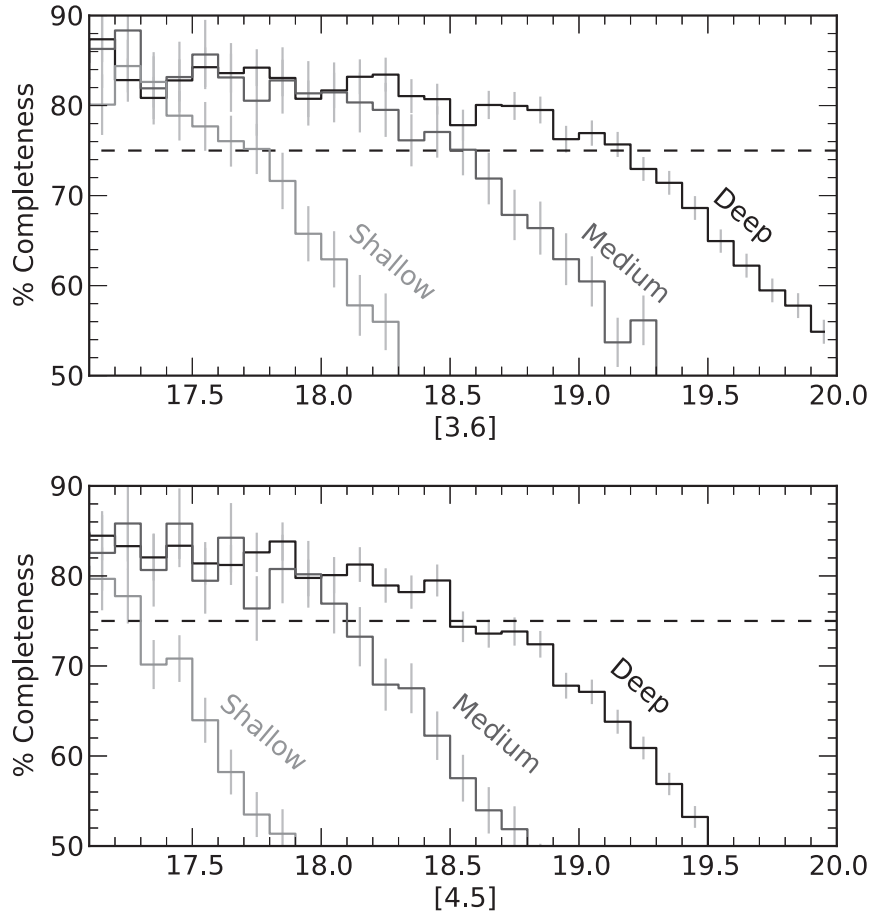

Figure 6. Average completeness curves for the shallow, medium, and deep epoch 1 data. For the deeper, combined-epoch photometry, the completeness limits are approximately $0.5 \mathrm{mag}$ fainter. The dashed line marks $75 \%$ completeness. These curves were derived for off regions (Section 4.4) and reflect only completeness due to sensitivity. These curves exclude galaxies that suffer from additional crowding; for those galaxies, the curves have similar shapes, shifted toward the brighter magnitudes listed in Table 3 .
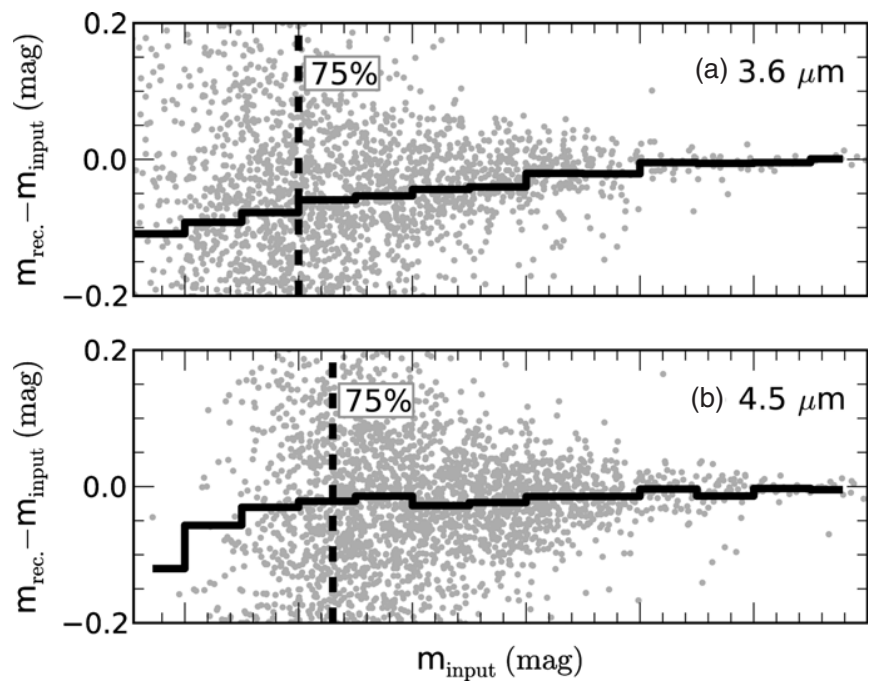

Figure 7. Difference between the input stellar magnitudes and the recovered stellar magnitudes from the artificial star tests. Three galaxies with $t_{\exp }=$ $1080 \mathrm{~s}$ are shown here. The $75 \%$ completeness limit is shown as a dashed line, and the solid black line shows the mean magnitude difference within $0.5 \mathrm{mag}$ bins, excluding sources outside $3 \sigma$. The magnitudes in the published catalogs are corrected for the bias shown here.

0.5 mag fainter, resulting in near-complete photometry to the TRGB in all 50 galaxies.

\subsection{Crowding}

Stellar crowding affects the photometric completeness both in the centers of dense galaxies (intrinsic) and for galaxies near 
Table 4

Intrinsic Crowding Limits

\begin{tabular}{lccc}
\hline \hline Galaxy & $\begin{array}{c}m_{3.6} \\
=m_{75 \%}\end{array}$ & $\begin{array}{c}M_{3.6} \\
=-6 \mathrm{mag}\end{array}$ & $\begin{array}{c}M_{3.6} \\
=-8 \mathrm{mag}\end{array}$ \\
\hline IC 10 & $R \approx 5^{\prime}$ & $\ldots$ & $R \approx 1^{\prime}$ \\
NGC 147 & $R \approx 4^{\prime}$ & $R \approx 4^{\prime}$ & All $R$ \\
NGC 185 & $R \approx 4^{\prime}$ & $R \approx 3^{\prime}$ & All $R$ \\
\hline
\end{tabular}

Notes. The radii $(R)$ where $75 \%$ completeness is reached for the given absolute magnitudes in galaxies that suffer from intrinsic crowding in their centers. Crowding was measured at radius intervals of $1^{\prime}$. Note that NGC 147 is elongated (ellipticity $\epsilon=0.41 \pm 0.02$; McConnachie 2012), so photometry is complete at smaller radii along the minor axis.

Table 5

GSC Catalog Description

\begin{tabular}{ll}
\hline \hline Column & \\
\hline 1 & Galaxy Name \\
2 & Point-source ID number \\
3 & Point-source name; IAU convention \\
$4-5$ & R.A. (degrees), decl. (degrees); J(2000) \\
$6-7$ & R.A. (h:m:s), decl. $\left({ }^{\circ}:{ }^{\prime}:{ }^{\prime \prime}\right) ; \mathrm{J}(2000)$ \\
$8-13$ & $3.6 \mu \mathrm{m}$ mag and uncertainty for each epoch and combined epochs \\
$14-19$ & 4.5 $\mu \mathrm{m}$ mag and uncertainty for each epoch and combined epochs \\
$20-22$ & DAOphot $S_{3.6}$ values for each epoch and combined epochs \\
$23-25$ & DAOphot $S_{4.5}$ values for each epoch and combined epochs \\
$26-28$ & DAOphot $\chi_{3.6}$ values for each epoch and combined epochs \\
$29-31$ & DAOphot $\chi_{4.5}$ values for each epoch and combined epochs \\
\hline
\end{tabular}

Notes. The catalog is available for download via MAST, IRSA, and VizieR. The full catalog is also available and includes the flag described in Section 5.1.

the Galactic plane, where foreground stars from the Milky Way increase the stellar density (extrinsic; Figure 1).

We compute the photometric completeness as a function of radius to measure crowding from stars within the galaxies themselves. For most DUSTiNGS galaxies, internal crowding does not significantly affect the photometry. WLM and Sextans A show only slight crowding within $1^{\prime}$ of the their centers, affecting the photometric completeness by $\lesssim 0.2 \mathrm{mag}$ at $3.6 \mu \mathrm{m}$. Severe crowding is evident for IC 10, NGC 147, and NGC 185. Table 4 lists the radius where the photometry becomes $75 \%$ complete at absolute magnitudes of $M_{3.6}=-6$ and -8 mag, which are the limits used to identify AGB candidates in Section 6. IC 10 is the only galaxy for which the number of $x$-AGB (Section 1.2) candidates should be considered a lower limit.

All galaxies residing well above or below the Galactic plane show similar completeness limits, but the $75 \%$ completeness limit rapidly increases in brightness as the distance from the Galactic plane decreases. IC 10 and And VII have the smallest Galactic latitudes and are the most affected by foreground stars (Figure 1). Sag DIG has a higher Galactic latitude but its longitude places it near the Galactic bulge. The completeness limits for galaxies affected by extrinsic crowding are listed in Table 3.

\section{DESCRIPTION OF THE CATALOG}

The final Vega magnitudes of the high-quality point sources are reported in the DUSTiNGS “Good"-Source Catalog (GSC), which is described in Table 5 and is available to download from MAST, ${ }^{14}$ VizieR, ${ }^{16}$ and IRSA. ${ }^{15}$ To construct the GSC, we culled the full photometric catalogs using the sharpness
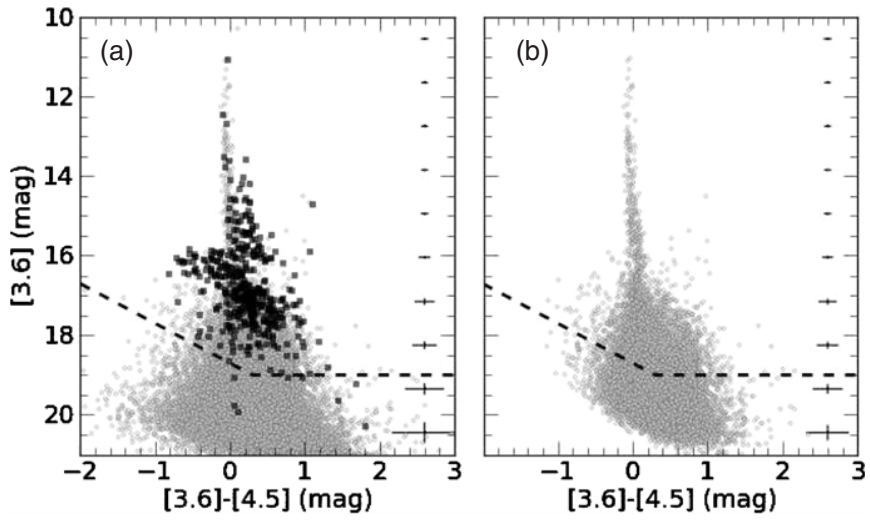

Figure 8. CMDs for And I showing (a) the full catalog and (b): the goodsource catalog. In the full catalog, marginally resolved sources are marked by darker points (Section 5.1). The [3.6]-[4.5] colors of extended sources can be artificially red or blue because fluxes may be extracted from the individual frames for one wavelength and from the mosaics for the other wavelength. The dashed line marks the $75 \%$ completeness limit. Mean photometric uncertainties are shown on the right of each panel.

$(S)$ and chi $(\chi)$ parameters returned by DAOphot. To eliminate artifacts and extended objects, the sharpness value is restricted to $-0.3<\left\langle S_{\lambda}\right\rangle<0.3$. The $\chi$ parameter is a measure of the rms of the residuals and is restricted to $\left\langle\chi_{\lambda}\right\rangle<5$ for sources measured from the $\mathrm{cBCD}$ frames and to $\left\langle\chi_{\lambda}\right\rangle<2$ for those measured from the mosaics. In addition, the GSC includes only sources detected above the $4 \sigma$ level and below the saturation limit and is restricted to sources that meet these criteria at both 3.6 and $4.5 \mu \mathrm{m}$. Figure 8 shows an example color-magnitude diagram (CMD) of the full catalog compared with the GSC. The CMDs using the GSC for all targeted galaxies are presented in Figure 12.

\subsection{Marginally Resolved Extended Sources}

Extended sources that are unresolved or marginally resolved in the individual cBCD frames are more strongly resolved in the subsampled mosaic. There are several sources measured from the $\mathrm{cBCD}$ frames that therefore meet the sharpness criteria for the GSC, but would fail the same criteria if measured on the mosaic. Because these sources are extended, PSF photometry is inappropriate and can result in large uncertainties; the PSFderived magnitude measured on the $\mathrm{CBCD}$ frames can differ from that measured on mosaic by $0.2-1$ mag. For sources near the transition magnitude where the individual-frame photometry and the mosaic photometry were combined (Section 4.1), this results in artificially blue or red colors (dark points in Figure 8) if stars were measured on the $\mathrm{cBCD}$ frames for one wavelength and on the mosaics for the other. These sources are easily identified via a mean sharpness value created by combining $S_{\lambda}$ measured by DAOphot for all measured channels and epochs from both the cBCD frames and mosaics. This combined sharpness parameter is larger for marginally resolved sources than for the true point sources at a given magnitude.

Removing these sources from the GSC significantly decreases the contamination from background sources brighter than $\approx 17 \mathrm{mag}$ and allows for a more accurate selection of stars belonging to the target galaxies. We do not remove these sources from the full catalog because we cannot rule out the possibility that they are indeed galaxy members (e.g., star clusters). However, the PSF-derived magnitudes for these sources are unreliable, so we include only their positions in the full catalog and recommend aperture photometry for accurate fluxes. 


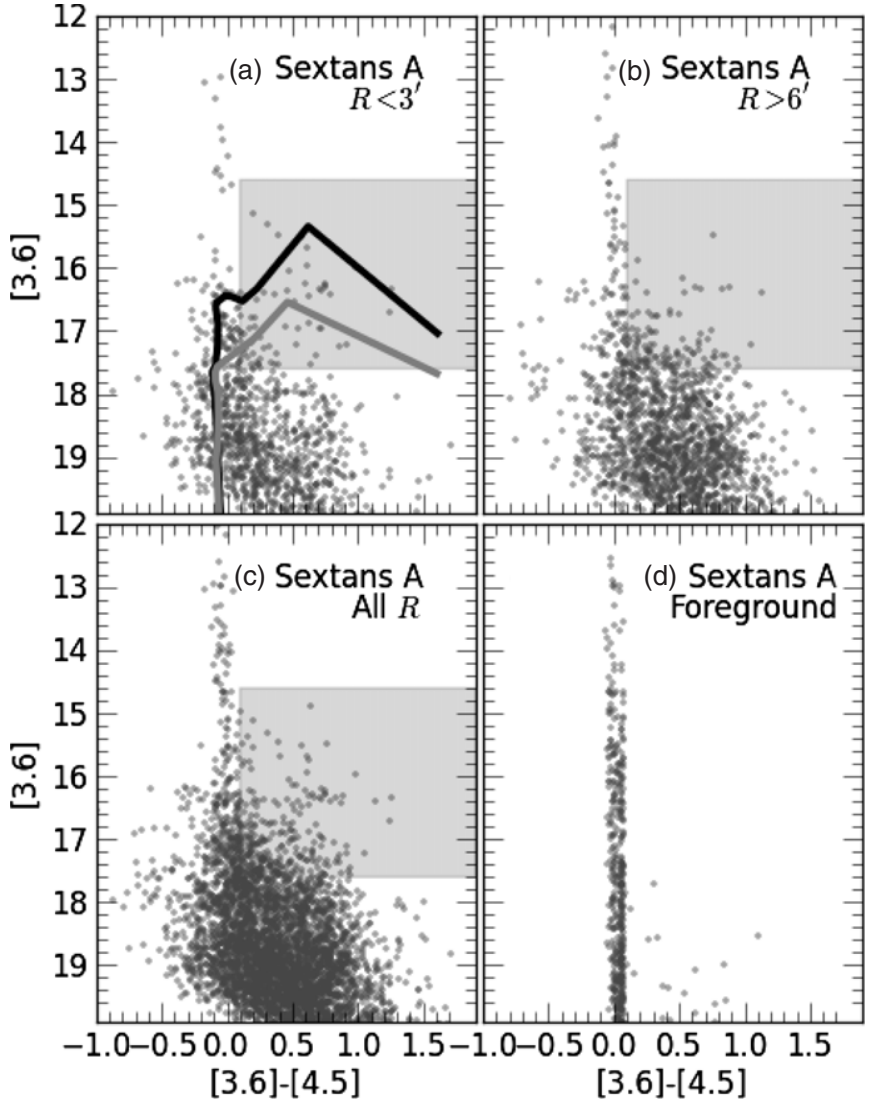

Figure 9. Epoch $1 \mathrm{CMD}$ for Sextans A, showing (a) sources within $3^{\prime}$, (b) sources beyond $6^{\prime},(\mathrm{c})$ the entire coverage, and (d) foreground simulation for the full spatial coverage (Table 2) from TRILEGAL. The shaded region shows the approximate location of $\mathrm{x}-\mathrm{AGB}$ stars, based on their position on the same CMD in the Magellanic Clouds (Section 6.1.2; Blum et al. 2006; Bolatto et al. 2007; Boyer et al. 2011). The half-light radius for Sextans A is 2'.47, with an ellipticity of only 0.17 (Figure 13; McConnachie 2012). In panel (a), the dark and light solid lines are $400 \mathrm{Myr}$ and $1 \mathrm{Gyr}$ isochrones, respectively, from Marigo et al. (2008).

\subsection{Extinction}

We have not corrected for extinction in the photometric catalogs. With the exception of IC 10, all DUSTiNGS galaxies show $\mathrm{E}(B-V)<0.2 \mathrm{mag}$ (McConnachie 2012). At 3.6 and $4.5 \mu \mathrm{m}$, this level of extinction results in a change in magnitude that is less than the photometric uncertainties $\left(A_{3.6}<0.03 \mathrm{mag}\right.$ and $A_{4.5}<0.02 \mathrm{mag}$ ).

IC 10 has the smallest Galactic latitude, and thus the highest level of extinction at $\mathrm{E}(B-V)=1.6 \mathrm{mag}$. Correcting the IRAC magnitudes for extinction would result in a magnitude decrease of $\sim 0.2 \mathrm{mag}$. However, the change in color due to extinction is still well below the photometric uncertainties with $\Delta\left(m_{3.6}-m_{4.5}\right)<0.04$ mag.

\subsection{Background and Foreground Contamination}

The DUSTiNGS field of view is large enough to provide a robust estimate of the foreground and background sources. Figure 9(c) shows the epoch 1 CMD for Sextans A, one of the more distant DUSTiNGS galaxies $\left(r_{\mathrm{h}}=2\right.$ '.47; also see Figure 13). To demonstrate a CMD with minimal contamination from nonmembers and a CMD that is dominated by background and foreground, we also show the CMDs of inner and outer regions of the Sextans A coverage in Figures 9(a) and (b). We show an estimate of the foreground in panel $d$, simulated with
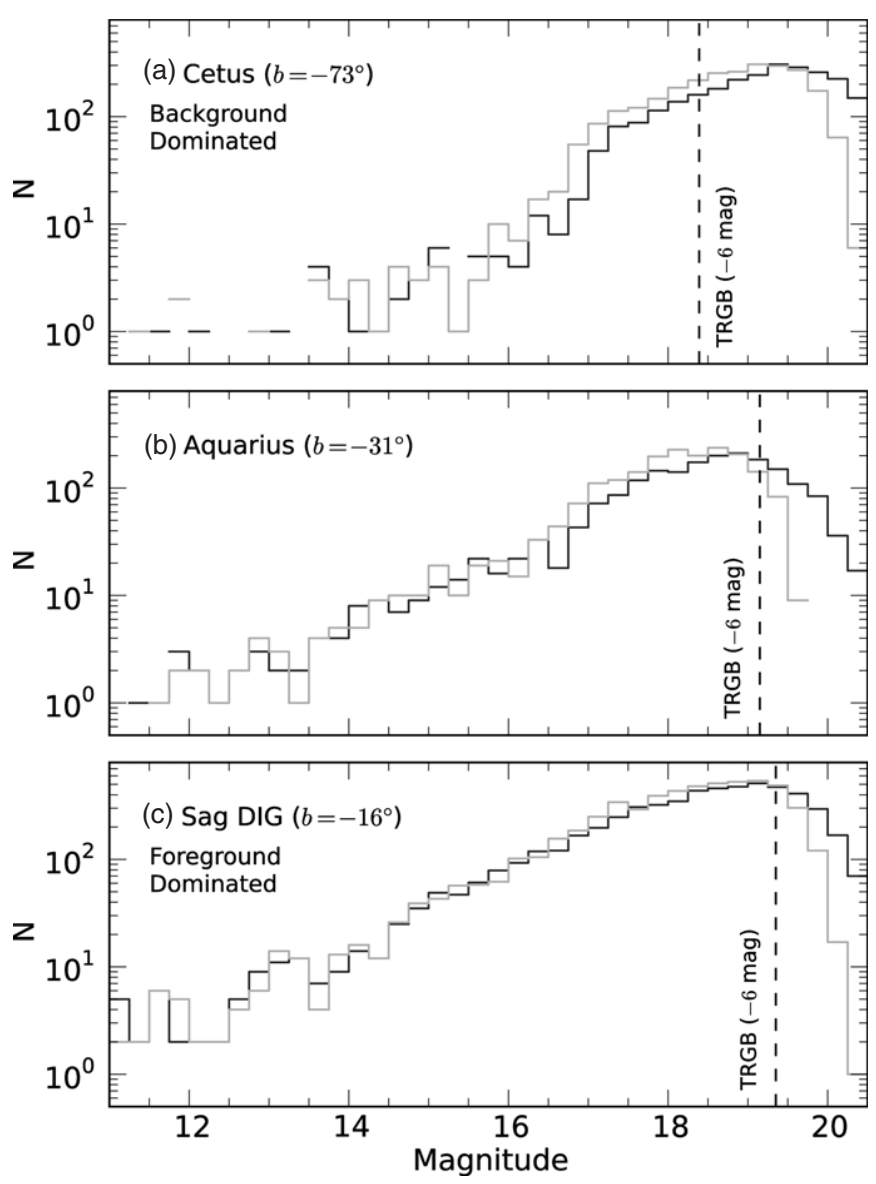

Figure 10. Luminosity functions for (a) Cetus, (b) Aquarius, and (c) Sag DIG. The black and gray lines are the $3.6 \mu \mathrm{m}$ and $4.5 \mu \mathrm{m}$ luminosity functions, respectively. Because Cetus lies far from the Galactic plane, its luminosity function is dominated by red background sources, mostly fainter than $17 \mathrm{mag}$. Sag DIG $\left(b=-16^{\circ}\right)$ is dominated by foreground from the Galactic bulge. In all panels, the expected TRGB $\left(M_{[3.6]} \approx-6 \mathrm{mag}\right)$ is marked with a dashed line.

the TRILEGAL stellar population synthesis code (Girardi et al. 2005). The difficulty in distinguishing between dusty stars with [3.6]-[4.5] $>0.1 \mathrm{mag}$ and $M_{3.6}<-8 \mathrm{mag}$ and unresolved background sources in the same color-magnitude space (shaded region of Figure 9) is clear when comparing panels (a) and (b). Less dusty member stars (with [3.6]-[4.5] $\approx 0$ mag) are also difficult to identify due to confusion with both background and foreground sources. Because AGB stars and some massive stars are variable, the dual-epoch DUSTiNGS observations are crucial for identifying individual member stars (Paper II).

In galaxies with a large intermediate-aged stellar population, a branch of $\mathrm{x}$-AGB stars (Section 6.1.2) that follows the isochrones shown in Figure 9(a) is easily identifiable in the CMD (Figure 12). This feature is clearly visible in only a handful of the DUSTiNGS galaxies: IC 10, IC 1613, NGC 147, and NGC 185. Even in other star-forming DUSTiNGS galaxies (e.g., WLM, Sag DIG, Sextans A, Sextans B, and Pegasus dIrr), this branch is not easily distinguished from background sources.

\subsection{Luminosity Functions}

Most of the DUSTiNGS galaxies have much smaller angular sizes than the field of view (Table 1) and the recovered photometry is therefore dominated by foreground and/or background sources. We demonstrate this in Figure 10 for Cetus, Aquarius, and Sag DIG. Cetus is far from the Galactic Plane $\left(b=-73^{\circ}\right.$; 

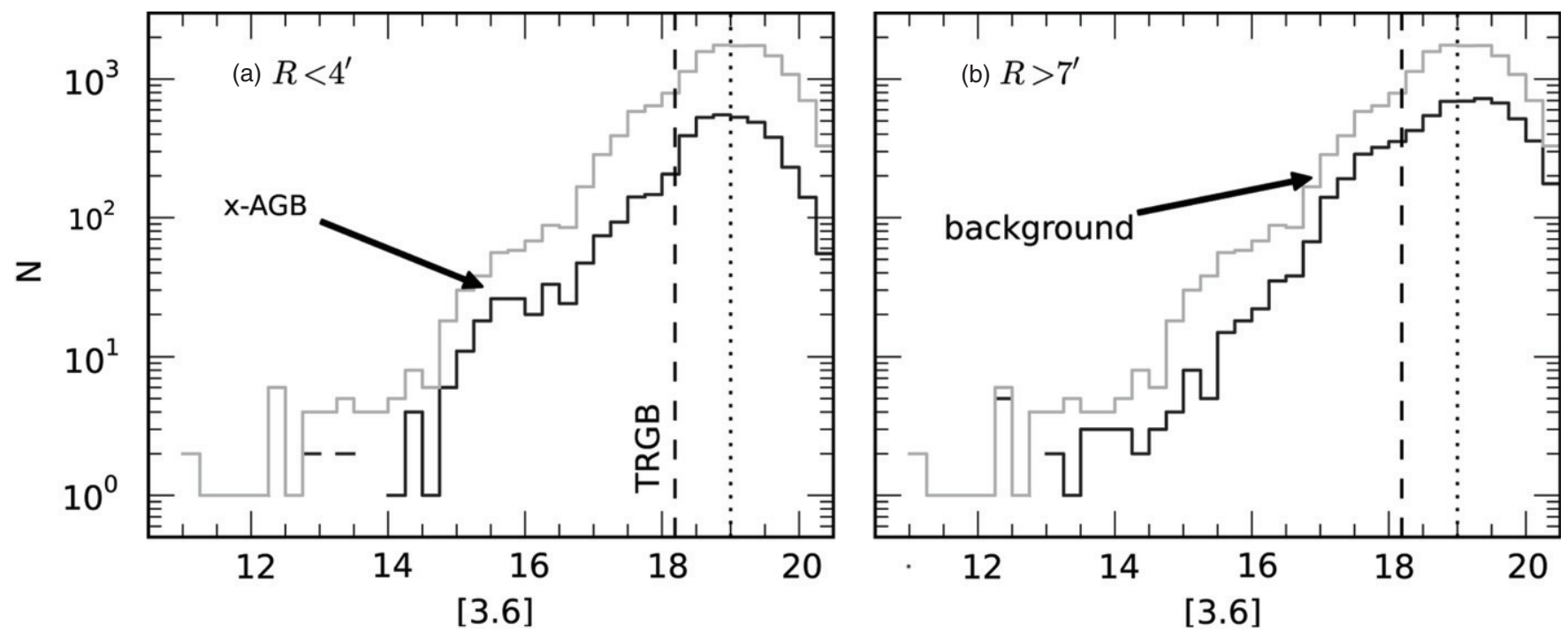

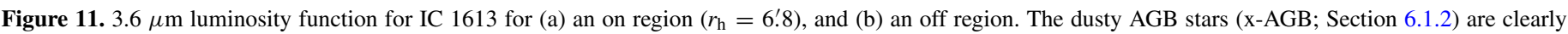

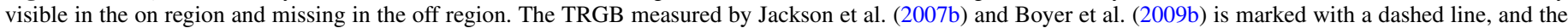
$75 \%$ completeness limit is marked with a dotted line. The gray histogram in each panel shows the luminosity function for the entire field of view.

Figure 1), and so is dominated by red background sources. This causes the $4.5 \mu \mathrm{m}$ luminosity function to appear brighter than the $3.6 \mu \mathrm{m}$ luminosity function. It also results in a sharp drop-off near $17 \mathrm{mag}$, because most of the brighter background galaxies have been eliminated from the GSC (Section 5.1). At the other extreme, Sag DIG is along a line of sight near the Galactic Bulge $\left(b=-16^{\circ}\right)$. Foreground therefore dominates its luminosity function and since these stars have colors near zero, the luminosity function is nearly the same at both wavelengths. Aquarius is at an intermediate latitude and shows the signatures of both foreground and background sources.

In Figure 11, we show the $3.6 \mu \mathrm{m}$ luminosity function for on and off regions toward IC 1613, which is known to harbor a large intermediate-aged stellar population (e.g., Skillman et al. 2014). At $R<4^{\prime}$, the TRGB and a feature attributed to X-AGB stars (Section 6.1.2) are visible. These same features are visible in other galaxies with large AGB populations. For $R>7^{\prime}$, a feature attributable to background sources is visible from $17<m_{[3.6]}<18 \mathrm{mag}$.

\section{THE IR STELLAR POPULATIONS}

We cannot separate member stars from background/ foreground sources with only the DUSTiNGS wavelengths. Therefore, we statistically subtract foreground and background sources to estimate the sizes of the TP-AGB $\left(N_{\mathrm{TRGB}}\right)$ and X-AGB $\left(N_{\mathrm{xAGB}}\right)$ populations. In Paper II, we use the twoepoch variability information to identify a subset of individual AGB stars.

\subsection{Stellar Classification \\ 6.1.1. AGB Stars $\left(N_{T R G B}\right)$}

We classify all sources brighter than the TRGB as TP-AGB candidates, and assume that the TRGB lies at $M_{3.6}=-6$ mag. The TRGB is unknown for most of the DUSTiNGS galaxies, but Jackson et al. (2007a, 2007b) and Boyer et al. (2009b) find that it is $-6.6<M_{3.6}<-6$ mag for 8 of the DUSTiNGS dIrr galaxies. Using the Padova stellar evolution models (Marigo et al. 2008, 2013), Bruzual et al. (2013) created simple stellar population models of the Magellanic Clouds. They find that $>90 \%$ of the TP-AGB stars are brighter than the TRGB (G. Bruzual 2013, private communication), so using the TRGB cutoff ensures that most of TP-AGB stars are included here. We apply no additional color cuts to the general TP-AGB classification.

The photometry is not $100 \%$ complete at the assumed TRGB for most of the DUSTiNGS galaxies. We therefore include a completeness-corrected value of $N_{\text {TRGB }}$ in Table 6 (see below). We do not, however, correct for intrinsic crowding, which affects only the inner region of IC 10, NGC 147, and NGC 185 (Table 4).

The parameter $N_{\text {TRGB }}$ includes AGB stars, massive young stars, and massive evolved stars. Without data at shorter wavelengths, it is impossible to know what fraction of $N_{\mathrm{TRGB}}$ is indeed AGB stars. In the Magellanic Clouds, AGB stars account for $38 \%$ (LMC) to $43 \%$ (SMC) of the stars brighter than -6 mag (derived from SAGE data after subtraction of foreground sources; Boyer et al. 2011). For galaxies with recent star formation (i.e., the dIrr galaxies and NGC 185 and NGC 147), we expect that the number of AGB candidates is $\gtrsim 0.3 N_{\mathrm{TRGB}}$, based on the LMC and SMC results. In the quiescent galaxies (i.e., most of the $\mathrm{dSph}$ galaxies), we can be confident that all, or nearly all, of $N_{\text {TRGB }}$ are AGB candidates. Stars more massive than $M \gtrsim 8 M_{\odot}$ will not go through the AGB phase, so unless star formation has occurred in the last $50 \mathrm{Myr}$, there will not be contamination from massive stars in $N_{\mathrm{TRGB}}$.

$$
\text { 6.1.2. } x \text {-AGB Stars }\left(N_{x A G B}\right)
$$

The $\mathrm{x}$-AGB stars are a very dusty subset of the general TPAGB population $\left(N_{\mathrm{TRGB}}\right.$ includes $\left.N_{\mathrm{xAGB}}\right)$. More than $90 \%$ of TP-AGB stars with [3.6]-[4.5] $>0.1 \mathrm{mag}$ and $M_{3.6}=-8 \mathrm{mag}$ in the Magellanic Clouds are classified as X-AGB stars by Blum et al. (2006) and Boyer et al. (2011), and we use the same criteria to classify them here. We emphasize that the $\mathrm{x}$-AGB label is not synonymous with dust-producing or exclusive; TP-AGB stars with bluer colors may be producing dust, though at a smaller rate (Riebel et al. 2012; Boyer et al. 2012). This x-AGB star classification is based solely on the observed IR color, and it roughly corresponds to AGB sources that are in the superwind 
Table 6

AGB Population Size

\begin{tabular}{|c|c|c|c|c|c|c|c|c|c|}
\hline \multirow[b]{2}{*}{ Galaxy } & \multicolumn{2}{|c|}{ Raw Counts } & \multicolumn{2}{|c|}{ Corrected } & \multirow[b]{2}{*}{ Galaxy } & \multicolumn{2}{|c|}{ Raw Counts } & \multicolumn{2}{|c|}{ Corrected } \\
\hline & $N_{\text {TRGB }}{ }^{\mathrm{a}}$ & $N_{\mathrm{xAGB}}{ }^{\mathrm{b}}$ & $N_{\text {TRGB }}{ }^{\mathrm{a}}$ & $N_{\mathrm{xAGB}}{ }^{\mathrm{b}}$ & & $N_{\mathrm{TRGB}}{ }^{\mathrm{a}}$ & $N_{\mathrm{xAGB}}{ }^{\mathrm{b}}$ & $N_{\mathrm{TRGB}}{ }^{\mathrm{a}}$ & $N_{\mathrm{xAGB}}^{\mathrm{b}}$ \\
\hline And I & $168 \pm 33$ & $\leqslant 7$ & $197 \pm 36$ & $\leqslant 8$ & Coma & $\leqslant 3$ & 0 & $\leqslant 3$ & 0 \\
\hline And II & $73 \pm 31$ & $9 \pm 4$ & $86 \pm 34$ & $11 \pm 4$ & CVn II & $\leqslant 5$ & 0 & $\leqslant 5$ & 0 \\
\hline And III & $136 \pm 31$ & $\leqslant 6$ & $163 \pm 34$ & $\leqslant 7$ & Hercules & $20 \pm 9$ & 0 & $24 \pm 10$ & 0 \\
\hline And V & $71 \pm 39$ & $\leqslant 8$ & $77 \pm 43$ & $\leqslant 8$ & IC $10^{\mathrm{c}}$ & $11,200 \pm 137$ & $516 \pm 23$ & $16,996 \pm 158$ & $597 \pm 25$ \\
\hline And VI & $160 \pm 30$ & $\leqslant 6$ & $190 \pm 33$ & $\leqslant 6$ & IC 1613 & $2224 \pm 85$ & $64 \pm 10$ & $2607 \pm 91$ & $67 \pm 11$ \\
\hline And VII & $506 \pm 48$ & $\leqslant 10$ & $628 \pm 54$ & $\leqslant 11$ & Leo A & $53 \pm 28$ & $\leqslant 5$ & $63 \pm 31$ & $\leqslant 5$ \\
\hline And IX & $\leqslant 44$ & $\leqslant 3$ & $\leqslant 47$ & $\leqslant 3$ & Leo IV & $\leqslant 6$ & 0 & $\leqslant 6$ & 0 \\
\hline And $X$ & $227 \pm 35$ & $\leqslant 6$ & $266 \pm 38$ & $\leqslant 6$ & Leo $\mathrm{T}$ & $32 \pm 12$ & $\leqslant 1$ & $36 \pm 13$ & $\leqslant 1$ \\
\hline And XI & $95 \pm 30$ & $\leqslant 7$ & $110 \pm 33$ & $\leqslant 7$ & Leo V & $\leqslant 5$ & 0 & $\leqslant 6$ & 0 \\
\hline And XII & $110 \pm 36$ & $\leqslant 9$ & $132 \pm 40$ & $\leqslant 9$ & LGS 3 & $\leqslant 34$ & $\leqslant 3$ & $\leqslant 36$ & $\leqslant 3$ \\
\hline And XIII & $119 \pm 31$ & $\leqslant 6$ & $146 \pm 34$ & $\leqslant 6$ & NGC $147^{c}$ & $4646 \pm 88$ & $109 \pm 12$ & $6342 \pm 100$ & $124 \pm 13$ \\
\hline And XIV & $50 \pm 29$ & $\leqslant 5$ & $58 \pm 31$ & $\leqslant 5$ & NGC $185^{\mathrm{c}}$ & $4119 \pm 78$ & $86 \pm 10$ & $5180 \pm 86$ & $99 \pm 11$ \\
\hline And XV & $46 \pm 26$ & $\leqslant 4$ & $55 \pm 29$ & $\leqslant 5$ & Pegasus & $742 \pm 54$ & $\leqslant 11$ & $882 \pm 58$ & $\leqslant 12$ \\
\hline And XVI & $40 \pm 30$ & $\leqslant 1$ & $46 \pm 21$ & $\leqslant 1$ & Phoenix & $61 \pm 16$ & $\leqslant 2$ & $68 \pm 17$ & $\leqslant 3$ \\
\hline And XVII & $128 \pm 38$ & $\leqslant 9$ & $150 \pm 41$ & $\leqslant 10$ & Pisces II & $\leqslant 9$ & 0 & $\leqslant 10$ & 0 \\
\hline And XVIII & $317 \pm 53$ & $\leqslant 24$ & $406 \pm 60$ & $\leqslant 26$ & Sag DIG & $829 \pm 79$ & $\leqslant 26$ & $1239 \pm 92$ & $\leqslant 29$ \\
\hline And XIX & $\leqslant 62$ & $\leqslant 9$ & $\leqslant 67$ & $\leqslant 9$ & Segue 1 & $\leqslant 1$ & 0 & $\leqslant 1$ & 0 \\
\hline And XX & $130 \pm 30$ & $\leqslant 7$ & $157 \pm 33$ & $\leqslant 8$ & Segue 2 & $\leqslant 2$ & 0 & $\leqslant 3$ & 0 \\
\hline And XXI & $116 \pm 39$ & $\leqslant 10$ & $135 \pm 43$ & $\leqslant 11$ & Segue 3 & $\leqslant 1$ & 0 & $\leqslant 2$ & 0 \\
\hline And XXII & $99 \pm 36$ & $\leqslant 8$ & $122 \pm 40$ & $\leqslant 9$ & Sextans A & $965 \pm 79$ & $\leqslant 34$ & $1230 \pm 88$ & $\leqslant 37$ \\
\hline Antlia & $204 \pm 48$ & $\leqslant 23$ & $260 \pm 54$ & $\leqslant 25$ & Sextans B & $1613 \pm 75$ & $77 \pm 20$ & $2118 \pm 86$ & $88 \pm 22$ \\
\hline Aquarius & $205 \pm 75$ & $\leqslant 14$ & $253 \pm 53$ & $\leqslant 15$ & Tucana & $150 \pm 35$ & $\leqslant 6$ & $183 \pm 38$ & $\leqslant 6$ \\
\hline Bootes I & $\leqslant 8$ & 0 & $\leqslant 8$ & 0 & UMa II & $\leqslant 2$ & 0 & $\leqslant 2$ & 0 \\
\hline Bootes II & 0 & 0 & 0 & 0 & Willman 1 & 0 & 0 & 0 & 0 \\
\hline Cetus & $140 \pm 29$ & $7 \pm 4$ & $166 \pm 31$ & $9 \pm 4$ & WLM & $1764 \pm 72$ & $59 \pm 12$ & $2077 \pm 78$ & $67 \pm 13$ \\
\hline
\end{tabular}

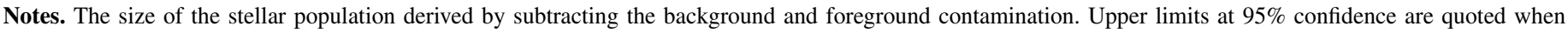

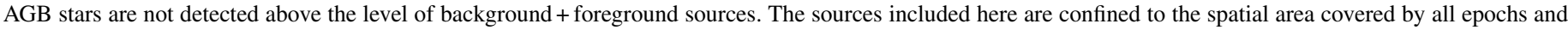
wavelengths (Table 2). We report both the raw counts and the counts corrected for photometric completeness (Section 4.4).

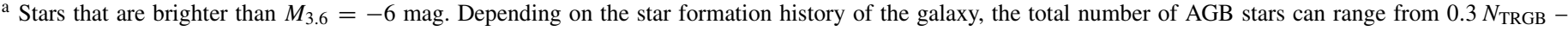
$N_{\text {TRGB }}$ (see text).

b xAGB stars are those brighter than $M_{3.6}=-8$ mag and redder than [3.6]-[4.5] $=0.1 \mathrm{mag}$.

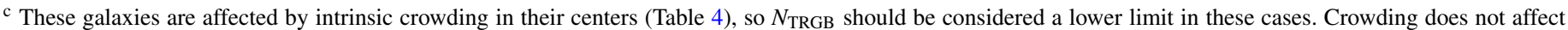
$N_{\text {xAGB }}$ except within the central $\approx 1^{\prime}$ region of IC 10 . We have not corrected numbers in this table for intrinsic crowding.

phase, when the mass-loss rate exceeds the nuclear-consumption rate and the dust-production rate can increase by more than a factor of 10 .

For galaxies observed with the longest total exposure times $\left(t_{\exp }=1080 \mathrm{~s}\right)$, the magnitude uncertainties for x-AGB stars is $\lesssim 0.04 \mathrm{mag}$ ( $1 \sigma$; Figure 4$)$, so a color of [3.6]-[4.5] $=0.1 \mathrm{mag}$ has a significance of $\gtrsim 2.5 \sigma$. Therefore, the $\mathrm{x}$-AGB class will include some sources that are not truly dusty and vice versa. For galaxies with shorter total exposure times $\left(t_{\exp }=60\right.$ $\mathrm{s}$ and $150 \mathrm{~s}$ ), the photometric uncertainties are larger and lie around $0.1 \mathrm{mag}$. In these cases, any infrared excess will have less significance. However, none of the galaxies with medium and shallow total exposure times show evidence for any sources redder than [3.6]-[4.5] $=0.1 \mathrm{mag}$, AGB or otherwise (Table 6).

$N_{\text {xAGB }}$ excludes most of the AGB stars with low mass-loss rates, massive red supergiant stars, and massive main-sequence stars. The result thus provides an estimate of the number of (mostly C-rich) X-AGB stars, with limited contamination from other source types (cf. Bonanos et al. 2010; Boyer et al. 2011; Sewiło et al. 2013).

We caution that the notation used for dusty AGB stars varies. For example, Gruendl et al. (2008) reserve the term "extreme AGB stars" for the rarest, dustiest stars with [3.6]-[4.5] $~$ $3 \mathrm{mag}$.

\subsection{Background/Foreground Source Subtraction}

Each DUSTiNGS galaxy was observed with a large field of view to assist in subtracting the contribution of background and foreground sources. To estimate $N_{\mathrm{TRGB}}$ and $N_{\mathrm{xAGB}}$, we first determine the distance from each galaxy center where the radial profile of point sources becomes flat and measure the density of sources with the relevant colors and magnitudes beyond this distance $\left(\Sigma_{\mathrm{N}}\right)$. We then subtract $\Sigma_{\mathrm{N}} \times$ coverage area (Table 2) from the total number of point sources to obtain $N_{\text {TRGB }}$ and $N_{\text {xAGB }}$.

In regions where the stellar density is high, background galaxies are undetectable. In these regions, we subtract only the foreground sources, which we estimate for the position of each target galaxy using the TRILEGAL population synthesis code (Girardi et al. 2005, see Figure 9). Table 6 lists the resulting AGB population sizes. The uncertainties in these numbers are derived from background-limited Poisson statistics. If the number of sources is below the $1.6 \sigma$ limit, we quote $95 \%$ confidence upper limits.

Table 6 includes both the raw values of $N_{\mathrm{TRGB}}$ and $N_{\mathrm{xAGB}}$ and values that have been corrected for photometric completeness using each galaxy's completeness curve (Figure 6 shows the mean completeness curve for each photometric depth). To make this correction, we first apply the completeness curve to the total 


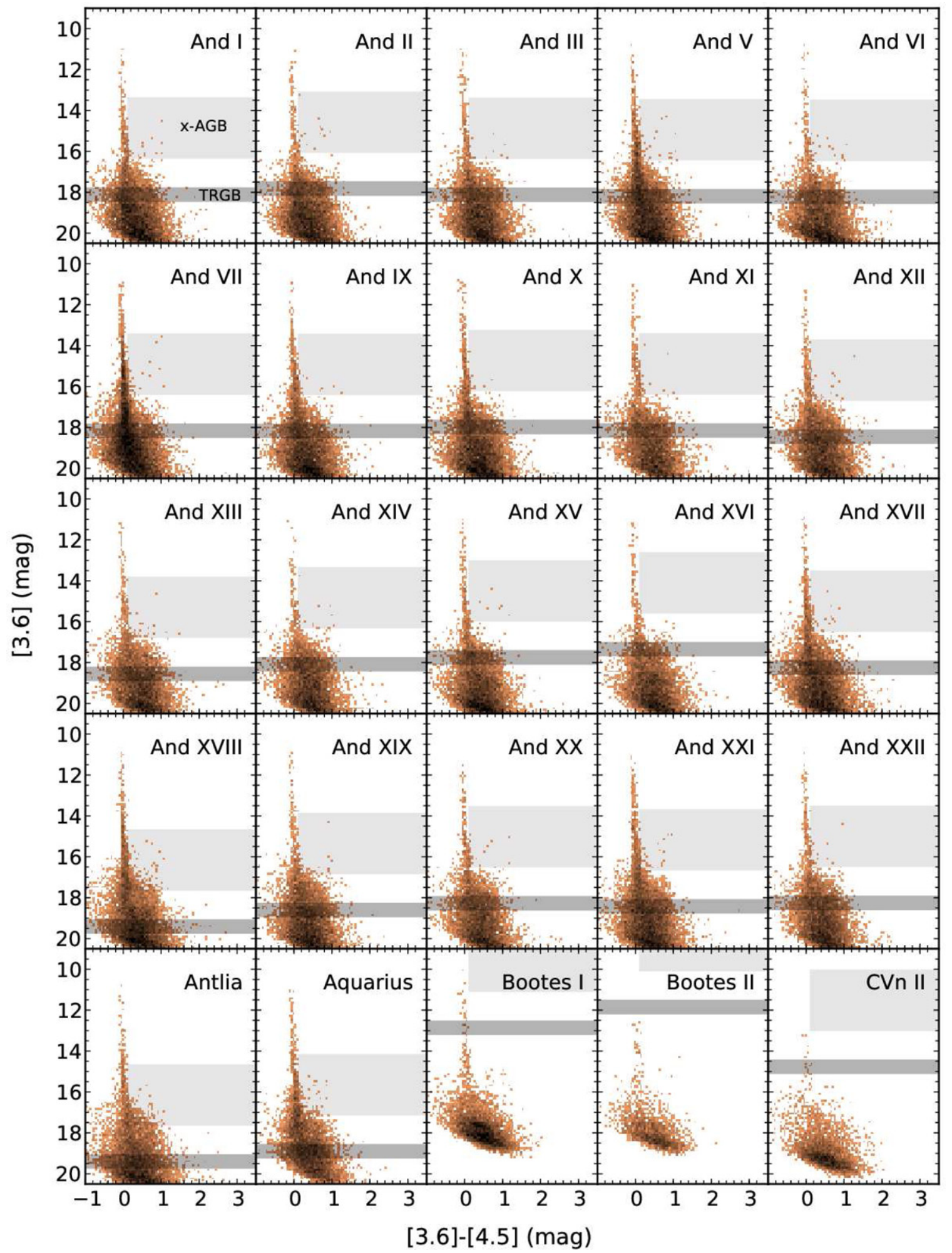

Figure 12. Color-magnitude diagrams of the GSC for each DUSTiNGS galaxy. Magnitudes shown here are derived from the two combined epochs. The dark shaded region marks the range of the possible TRGB and the light shaded region marks the approximate location of x-AGB stars.

number of counts and to $\Sigma_{\mathrm{N}}$ individually, then compute $N_{\mathrm{TRGB}}$ and $N_{\text {xAGB }}$ from those corrected values.

\subsection{Dust Production at Very Low Metallicity}

While most of the $x$-AGB stars in the DUSTiNGS sample are in the massive, more metal-rich galaxies (IC 10,
NGC 147 , NGC 185 , and WLM), we find $166 \pm 28 \mathrm{x}-\mathrm{AGB}$ stars at $[\mathrm{Fe} / \mathrm{H}] \approx-1.6$ and $9 \pm 4$ at $[\mathrm{Fe} / \mathrm{H}] \approx-1.9$ (Cetus). These are some of the most metal-poor dusty AGB stars known, and they are likely to be C-rich. AGB stars in the SMC with similar [3.6]-[4.5] colors have an average dust-production rate of $\log (\dot{D})=-8.7\left[M_{\odot} \mathrm{yr}^{-1}\right]$ (Boyer et al. 2012). 


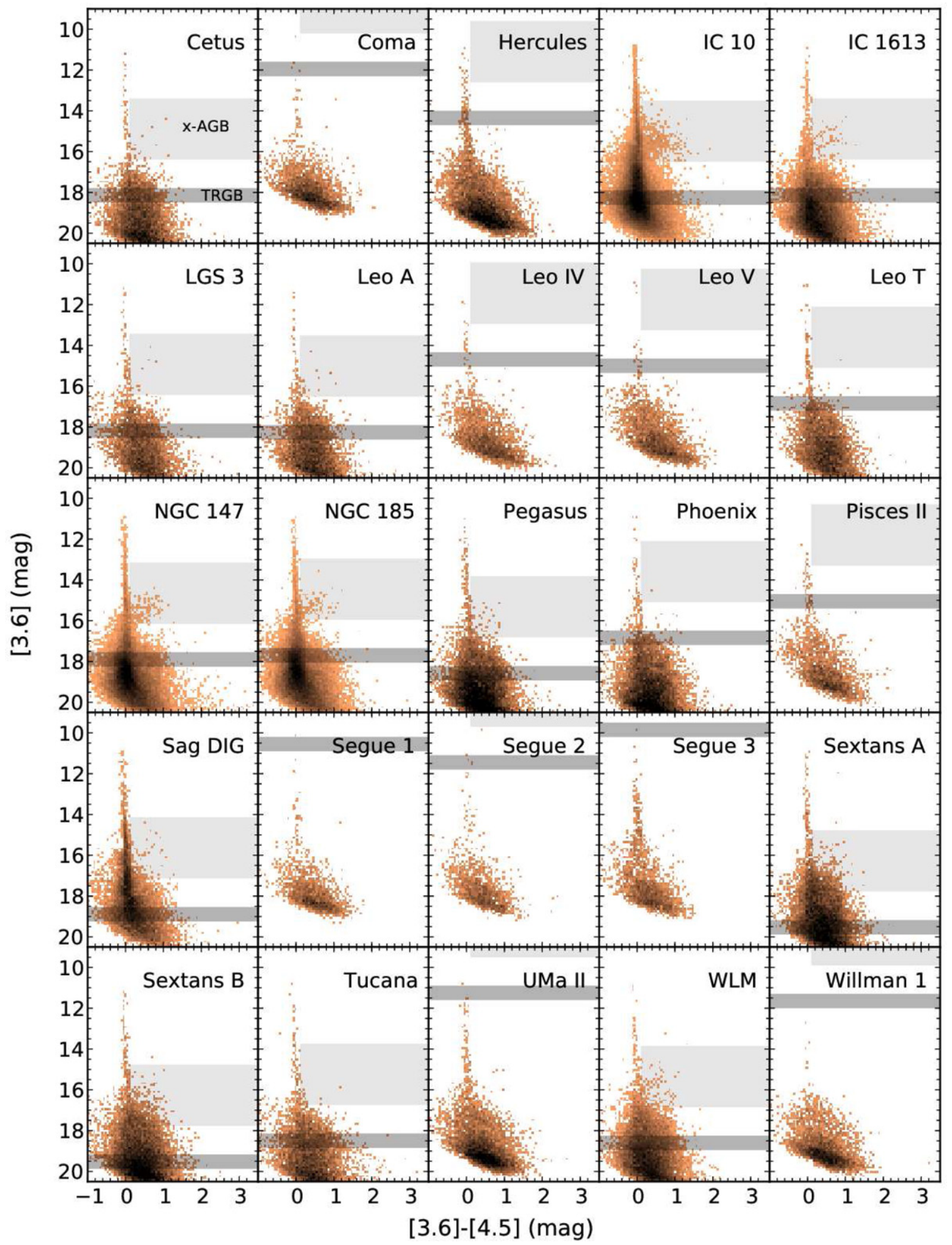

Figure 12. (Continued)

For galaxies with $[\mathrm{Fe} / \mathrm{H}]<-2$, we can quote only upper limits for the number of $\mathrm{x}-\mathrm{AGB}$ stars. On the other hand, we do detect $1645 \pm 240$ AGB stars with less dust in these metalpoor galaxies (And XI, And XII, And XIV, And XVI, Hercules, Leo T, Sag DIG). In the SMC, AGB stars at these colors have dust-production rates of $-10.7<\log (\dot{D})<-10.1\left[M_{\odot} \mathrm{yr}^{-1}\right]$. Because the $\mathrm{x}$-AGB population sizes are detected statistically, we can say little about the properties of the individual stars (e.g., their distribution in color and luminosity and their dustproduction rates). In Paper II, we identify a subset of the individual $\mathrm{x}-\mathrm{AGB}$ stars and further describe their characteristics.

\section{CONCLUSIONS}

DUSTiNGS is a 3.6 and $4.5 \mu \mathrm{m}$ photometric survey of 50 resolved dwarf galaxies within $1.5 \mathrm{Mpc}$ designed to detect dusty 

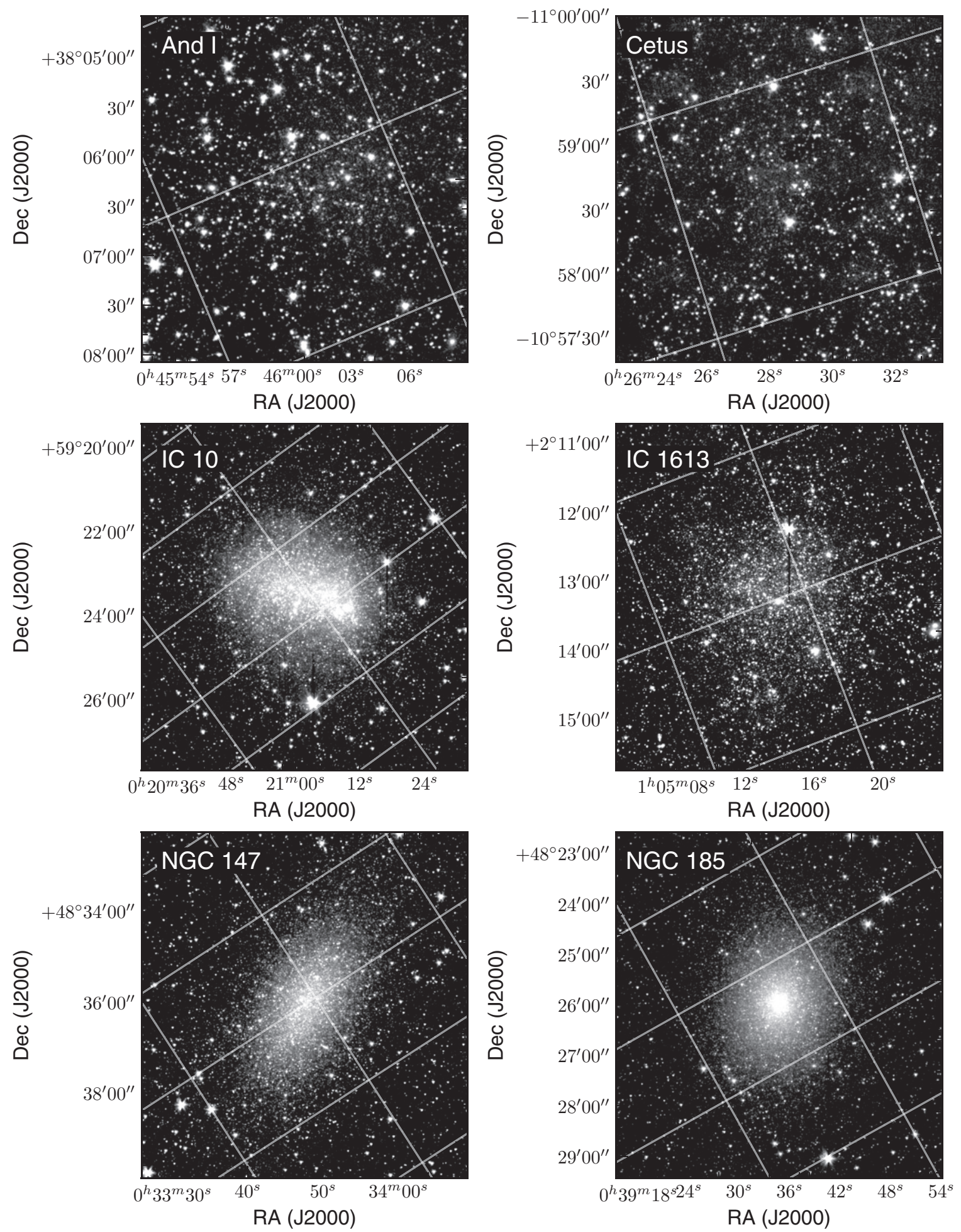

Figure 13. 3.6 $\mu \mathrm{m}$ epoch 1 mosaics for a subset of the DUSTiNGS galaxies.

evolved stars. The survey includes $37 \mathrm{dSph}$ galaxies, $8 \mathrm{dIrr}$ galaxies, and $5 \mathrm{dIrr} / \mathrm{dSph}$ transition-type galaxies. The large sample size allows for robust statistics on the short-lived, dustproducing phase. Each galaxy was observed over two epochs to aid in identifying variable AGB stars; Paper II presents the results of the variability analysis. Here, we describe the targets, the observing strategy, and the publicly available data products.

For all galaxies, the photometry is $>75 \%$ complete within the possible magnitude range of the TRGB with the exception of the inner regions of the most crowded galaxies: IC 10, NGC 147, and NGC 185. This completeness enables the detection of most of the AGB and massive evolved star populations. The photometric catalogs are publicly available at MAST, ${ }^{14}$ VizieR, ${ }^{16}$ and IRSA. ${ }^{15}$

Because it is difficult to distinguish dusty evolved stars from unresolved background objects at these wavelengths, the DUSTiNGS survey imaged an area larger than the halflight radius of each galaxy to allow for statistical subtraction 

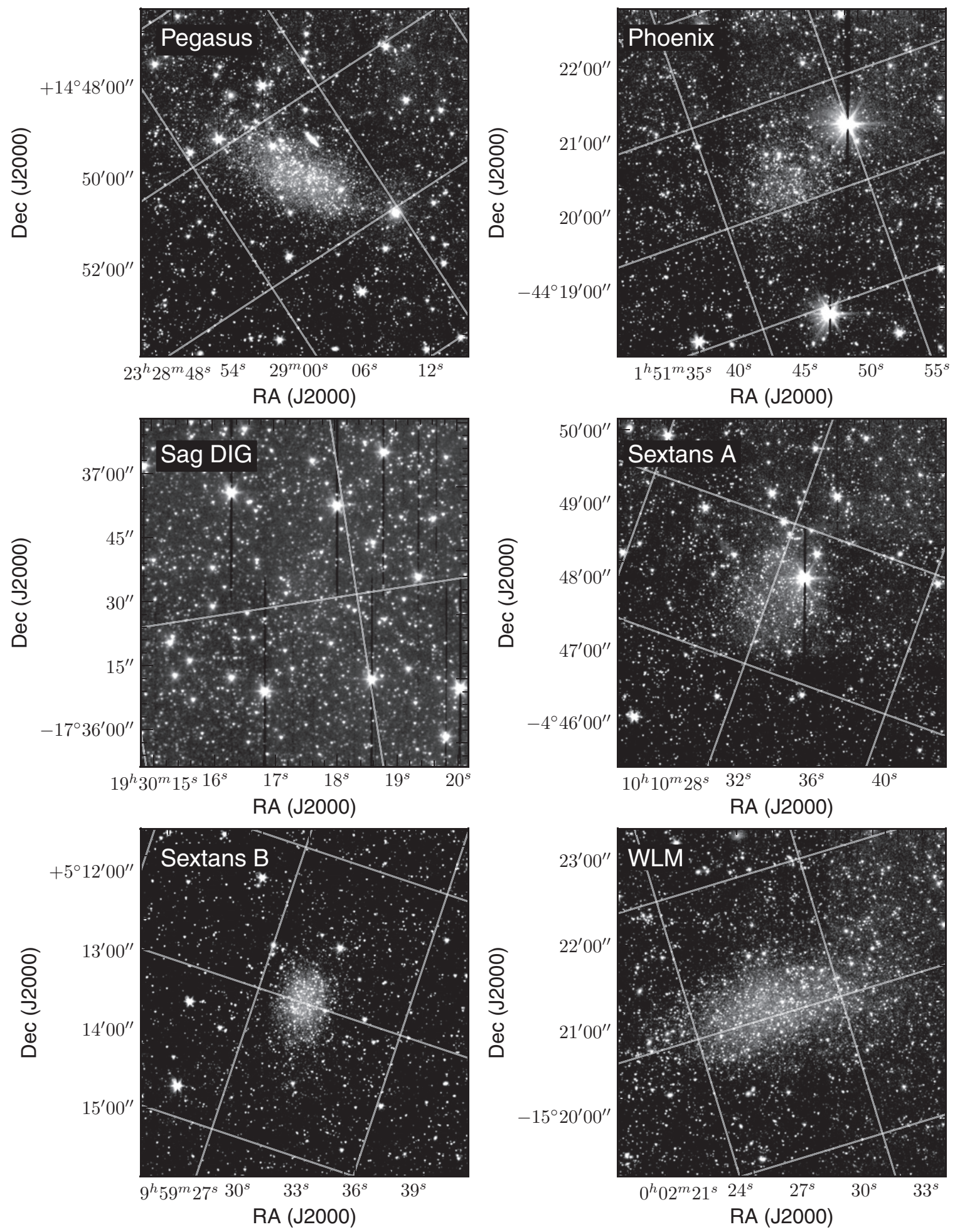

Figure 13. (Continued)

of foreground and background sources. We present here an estimate of the size of the stellar population brighter than the TRGB and the size of the dusty AGB star population. We find $1062 \pm 103$ "extreme" dusty AGB stars in 21 of the DUSTiNGS galaxies. For the remaining 29 DUSTiNGS galaxies we report $95 \%$ confidence upper limits.

Many thanks to Brian Babler for very helpful discussions about IRAC photometry. We also thank the referee for helpful comments. This work is supported by Spitzer via grant G080063 and by the NASA Astrophysics Data Analysis Program grant number N3-ADAP13-0058. M.L.B. is supported by the NASA Postdoctoral Program at the Goddard Space Flight Center, administered by ORAU through a contract with NASA. R.D.G. was supported by NASA and the United States Air Force. A.Z.B. acknowledges funding by the European Union (European Social Fund) and National Resources under the "ARISTEIA" action of the Operational Programme "Education and Lifelong Learning" 
in Greece. G.C.S. receives support from the NSF, award AST1108645 .

\section{APPENDIX A}

\section{COLOR-MAGNITUDE DIAGRAMS}

Figure 12 shows the DUSTiNGS CMDs from the GSCs. We show the combined epochs to demonstrate the maximum photometric depth. The dark shaded regions mark the range of the expected TRGB for each galaxy. The majority of TP-AGB stars are brighter than this limit. The light shaded regions mark the approximate location of $\mathrm{x}-\mathrm{AGB}$ stars.

\section{APPENDIX B \\ IMAGES}

Figure 13 show the $3.6 \mu \mathrm{m}$ epoch 1 mosaics for a subset of the DUSTiNGS galaxies. Galaxies not shown are low mass and have few sources above the TRGB. For these galaxies, it is difficult to see the galaxy among the background and foreground sources. We include Cetus as an example of a low-mass galaxy; see Figure 2 for an example of the imaging strategy.

\section{REFERENCES}

Babul, A., \& Rees, M. J. 1992, MNRAS, 255, 346

Bellazzini, M., Beccari, G., Fraternali, F., et al. 2014, A\&A, 566, A44

Belokurov, V., Walker, M. G., Evans, N. W., et al. 2010, ApJL, 712, L103

Benjamin, R. A., Churchwell, E., Babler, B. L., et al. 2003, PASP, 115, 953

Bernard, E. J., Monelli, M., Gallart, C., et al. 2010, ApJ, 712, 1259

Blum, R. D., Mould, J. R., Olsen, K. A., et al. 2006, AJ, 132, 2034

Bolatto, A. D., Simon, J. D., Stanimirović, S., et al. 2007, ApJ, 655, 212

Bonanos, A. Z., Lennon, D. J., Köhlinger, F., et al. 2010, AJ, 140, 416

Boyer, M. L., McDonald, I., van Loon, J. Th., et al. 2009a, ApJ, 705, 746

Boyer, M. L., McQuinn, K. B. W., Barmby, P., et al. 2014, ApJ, in press (Paper II)

Boyer, M. L., Skillman, E. D., van Loon, J. Th., Gehrz, R. D., \& Woodward,

C. E. 2009 b, ApJ, 697, 1993

Boyer, M. L., Srinivasan, S., Riebel, D., et al. 2012, ApJ, 748, 40

Boyer, M. L., Srinivasan, S., van Loon, J. Th., et al. 2011, AJ, 142, 103

Bressan, A., Marigo, P., Girardi, L., et al. 2012, MNRAS, 427, 127

Bruzual, G., Charlot, S., Lópezlira, R. G., et al. 2013, in IAU Symp. 295,

The Intriguing Life of Massive Galaxies, ed. D. Thomas, A. Pasquali, \& I.

Ferreras (Cambridge: Cambridge Univ. Press), 282

Chapman, S. C., Widrow, L., Collins, M. L. M., et al. 2013, MNRAS, 430, 37

Cole, A. A., Skillman, E. D., Tolstoy, E., et al. 2007, ApJL, 659, L17

Dekel, A., \& Silk, J. 1986, ApJ, 303, 39

Eddington, A. S. 1913, MNRAS, 73, 359

Efstathiou, G. 1992, MNRAS, 256, 43

Fadely, R., Willman, B., Geha, M., et al. 2011, AJ, 142, 88

Fazio, G. G., Hora, J. L., Allen, L. E., et al. 2004, ApJS, 154, 10

Fruchter, A. S., \& Hook, R. N. 2002, PASP, 114, 144

Gail, H.-P., Zhukovska, S. V., Hoppe, P., \& Trieloff, M. 2009, ApJ, 698, 1136

Gehrz, R. 1989, in IAU Symp. 135, Interstellar Dust, ed. L. J. Allamandola \& A. G. G. M. Tielens (Dordrecht: Kluwer), 445

Gehrz, R. D., Roellig, T. L., Werner, M. W., et al. 2007, RScI, 78, 011302

Girardi, L., Groenewegen, M. A. T., Hatziminaoglou, E., \& da Costa, L. 2005, A\&A, 436, 895

Gordon, K. D., Meixner, M., Meade, M. R., et al. 2011, AJ, 142, 102

Gordon, K. D., Roman-Duval, J., Bot, C., et al. 2014, ApJ, in press, arXiv: 1406.6066

Groenewegen, M. A. T. 2006, A\&A, 448, 181

Groenewegen, M. A. T., \& de Jong, T. 1993, A\&A, 267, 410
Groenewegen, M. A. T., Sloan, G. C., Soszyński, I., \& Petersen, E. A. 2009, A\&A, 506, 1277

Groenewegen, M. A. T., Wood, P. R., Sloan, G. C., et al. 2007, MNRAS, 376,313

Gruendl, R. A., Chu, Y.-H., Seale, J. P., et al. 2008, ApJL, 688, L9

Jackson, D. C., Skillman, E. D., Gehrz, R. D., Polomski, E., \& Woodward, C. E. $2007 \mathrm{a}, \mathrm{ApJ}, 656,818$

Jackson, D. C., Skillman, E. D., Gehrz, R. D., Polomski, E., \& Woodward, C. E. 2007b, ApJ, 667, 891

Jones, A. P., \& Nuth, J. A. 2011, A\&A, 530, A44

Karakas, A., \& Lattanzio, J. C. 2007, PASA, 24, 103

Kastner, J. H., Buchanan, C. L., Sargent, B., \& Forrest, W. J. 2006, ApJL, 638, L29

Kim, M., Kim, E., Hwang, N., et al. 2009, ApJ, 703, 816

Le Bertre, T. 1992, A\&AS, 94, 377

Le Bertre, T. 1993, A\&AS, 97, 729

Lee, H., Skillman, E. D., Cannon, J. M., et al. 2006, ApJ, 647, 970

Marigo, P., Bressan, A., Nanni, A., Girardi, L., \& Pumo, M. L. 2013, MNRAS, 434, 488

Marigo, P., Girardi, L., Bressan, A., et al. 2008, A\&A, 482, 883

Martin, N. F., McConnachie, A. W., Irwin, M., et al. 2009, ApJ, 705, 758

Mateo, M. L. 1998, ARA\&A, 36, 435

Matsuura, M., Barlow, M. J., Zijlstra, A. A., et al. 2009, MNRAS, 396, 918

Mattsson, L., Wahlin, R., Höfner, S., \& Eriksson, K. 2008, A\&A, 484, L5

Mayer, L., Governato, F., Colpi, M., et al. 2001, ApJL, 547, L123

Mayer, L., Mastropietro, C., Wadsley, J., Stadel, J., \& Moore, B. 2006, MNRAS, 369, 1021

McConnachie, A. W. 2012, AJ, 144, 4

McConnachie, A. W., Irwin, M. J., Ferguson, A. M. N., et al. 2005, MNRAS, 356,979

McDonald, I., Boyer, M. L., van Loon, J. T., et al. 2011a, ApJS, 193, 23

McDonald, I., van Loon, J. T., Sloan, G. C., et al. 2011b, MNRAS, 417, 20

McQuinn, K. B. W., Woodward, C. E., Willner, S. P., et al. 2007, ApJ, 664, 850

Meixner, M., Gordon, K. D., Indebetouw, R., et al. 2006, AJ, 132, 2268

Menzies, J., Feast, M., Whitelock, P., et al. 2008, MNRAS, 385, 1045

Miller, B. W., Dolphin, A. E., Lee, M. G., Kim, S. C., \& Hodge, P. 2001, ApJ, 562,713

Moffat, A. F. J. 1969, A\&A, 3, 455

Pimbblet, K. A., \& Couch, W. J. 2012, MNRAS, 419, 1153

Reach, W. T., Megeath, S. T., Cohen, M., et al. 2005, PASP, 117, 978

Riebel, D., Srinivasan, S., Sargent, B., \& Meixner, M. 2012, ApJ, 753, 71

Sand, D. J., Strader, J., Willman, B., et al. 2012, ApJ, 756, 79

Schneider, R., Valiante, R., Ventura, P., et al. 2014, MNRAS, 442, 1440

Sewiło, M., Carlson, L. R., Seale, J. P., et al. 2013, ApJ, 778, 15

Skillman, E. D., Côté, S., \& Miller, B. W. 2003, AJ, 125, 593

Skillman, E. D., Hidalgo, S. L., Weisz, D. R., et al. 2014, ApJ, 786, 44

Sloan, G. C., Kraemer, K. E., Wood, P. R., et al. 2008, ApJ, 686, 1056

Sloan, G. C., Matsunaga, N., Matsuura, M., et al. 2010, ApJ, 719, 1274

Sloan, G. C., Matsuura, M., Lagadec, E., et al. 2012, ApJ, 752, 140

Smith, N. 2014, ARA\&A, 52, 487

Smith, N., Gehrz, R. D., Hinz, P. M., et al. 2003, AJ, 125, 1458

Smith, N., Li, W., Foley, R. J., et al. 2007, ApJ, 666, 1116

Srinivasan, S., Meixner, M., Leitherer, C., et al. 2009, AJ, 137, 4810

Stetson, P. B. 1987, PASP, 99, 191

Tammann, G. A., Reindl, B., \& Sandage, A. 2011, A\&A, 531, A134

Tosi, M. 2003, Ap\&SS, 284, 651

van den Bergh, S. 1999, ApJL, 517, L97

van Loon, J. Th., Cohen, M., Oliveira, J. M., et al. 2008, A\&A, 487, 1055

Vijh, U. P., Meixner, M., Babler, B., et al. 2009, AJ, 137, 3139

Voors, R. H. M., Waters, L. B. F. M., de Koter, A., et al. 2000, A\&A, 356, 501

Wachter, A., Winters, J. M., Schröder, K.-P., \& Sedlmayr, E. 2008, A\&A, 486, 497

Watkins, L. L., Evans, N. W., \& van de Ven, G. 2013, MNRAS, 430, 971

Weisz, D. R., Dalcanton, J. J., Williams, B. F., et al. 2011, ApJ, 739, 5

Weisz, D. R., Dolphin, A. E., Skillman, E. D., et al. 2014, ApJ, 789, 147

Weisz, D. R., Zucker, D. B., Dolphin, A. E., et al. 2012, ApJ, 748, 88

Werner, M. W., Roellig, T. L., Low, F. J., et al. 2004, ApJS, 154, 1

Wright, E. L., Eisenhardt, P. R. M., Mainzer, A. K., et al. 2010, AJ, 140, 1868

Zhukovska, S., \& Henning, T. 2013, A\&A, 555, A99 